\title{
TFMC Tcs data: how do we compare conductor performance to the strand and what conclusions for ITER we may draw
}

\author{
N. Martovetsky
}

January 7, 2003

U.S. Department of Energy

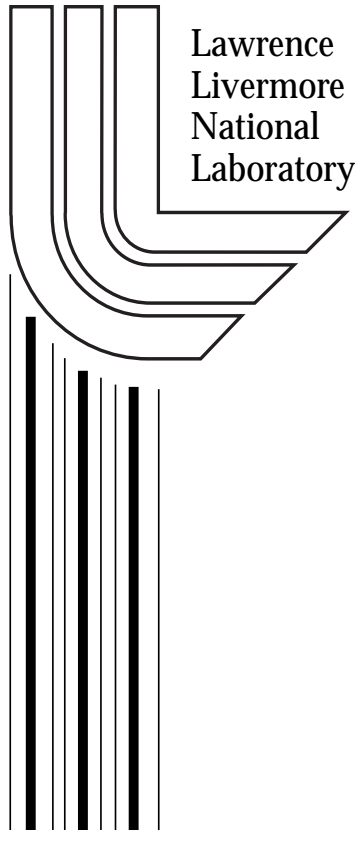




\section{DISCLAIMER}

This document was prepared as an account of work sponsored by an agency of the United States Government. Neither the United States Government nor the University of California nor any of their employees, makes any warranty, express or implied, or assumes any legal liability or responsibility for the accuracy, completeness, or usefulness of any information, apparatus, product, or process disclosed, or represents that its use would not infringe privately owned rights. Reference herein to any specific commercial product, process, or service by trade name, trademark, manufacturer, or otherwise, does not necessarily constitute or imply its endorsement, recommendation, or favoring by the United States Government or the University of California. The views and opinions of authors expressed herein do not necessarily state or reflect those of the United States Government or the University of California, and shall not be used for advertising or product endorsement purposes.

This work was performed under the auspices of the U. S. Department of Energy by the University of California, Lawrence Livermore National Laboratory under Contract No. W-7405-Eng-48.

This report has been reproduced directly from the best available copy.

Available electronically at http://www.doe.gov/bridge

Available for a processing fee to U.S. Department of Energy and its contractors in paper from

U.S. Department of Energy

Office of Scientific and Technical Information

P.O. Box 62

Oak Ridge, TN 37831-0062

Telephone: (865) 576-8401

Facsimile: (865) 576-5728

E-mail: reports@adonis.osti.gov

Available for the sale to the public from

U.S. Department of Commerce

National Technical Information Service 5285 Port Royal Road

Springfield, VA 22161

Telephone: (800) 553-6847

Facsimile: (703) 605-6900

E-mail: orders@ntis.fedworld.gov

Online ordering: http://www.ntis.gov/ordering.htm

OR

Lawrence Livermore National Laboratory

Technical Information Department's Digital Library

http://www.llnl.gov/tid/Library.html 


\section{Memo}

To: Distribution

From: Nicolai Martovetsky

CC:

Date: $1 / 07 / 03$

\section{Re: TFMC Tcs data: how do we compare conductor performance to the strand and what conclusions for ITER we may draw}

\section{Introduction}

This memo is to assess the TFMC test results and compare it with the strand performance. The TFMC is not an ideal object for studying performance of the CICC in a sense that the instrumentation priority was considered secondary to reliability and therefore a lot of assumptions and modeling need to be made to make the comparison against the LMI strand possible.

To compare the CICC performance to strand we need to know at least current in the strands, magnetic field and electric field distribution along the strands, temperature profile and strain distribution.

In the TFMC we have much less uniform magnetic field and less determined temperature than in the CSMC Inserts, so role of modeling is greater.

A code M\&M developed by Polito team (R. Zanino and L. Savoldi Richard) evaluates the temperature profile in the conductor. It includes heat transfer in joints, self-heating, heat transfer to the radial plates. From their model it follows [1] that the radial plates do not affect significantly the temperature in the conductor near the area with the peak magnetic field (which is 3-5 m away from the conductor entrance inside the TFMC winding pack. Unfortunately, there are no sensors in between the inlet and outlet to establish the validity of the code prediction, but different heater powers and outlet temperatures have good agreement with their code prediction, therefore we assume that their findings are correct. We will assume that the temperature in the TFMC is equal to the inlet temperature detected by the T712 sensor.

One of the difficult parts for the TFMC analysis that the magnetic field distribution is not uniform even longitudinally.

Due to significant current in the CICC the field distribution in the cable is never homogeneous.

In the CS Insert at $40 \mathrm{kA}$, the field difference between the high and low fields in the cross section was about $0.9 \mathrm{~T}$ [2]. In the TFMC at $80 \mathrm{kA}$, the difference is about $1.6 \mathrm{~T}$ [3]. The reason why the CS Insert has less field difference is higher transport current in the TFMC.

In addition to that transverse inhomogeneity, there is a strong inhomogeneity of the magnetic field along the length of the TFMC conductor.

In the CS Insert, about $40 \mathrm{~m}$ of conductor was sitting in a uniform field within several percent of the nominal one. The TFMC field distribution is shown in Fig. 1 taken from [3]. 


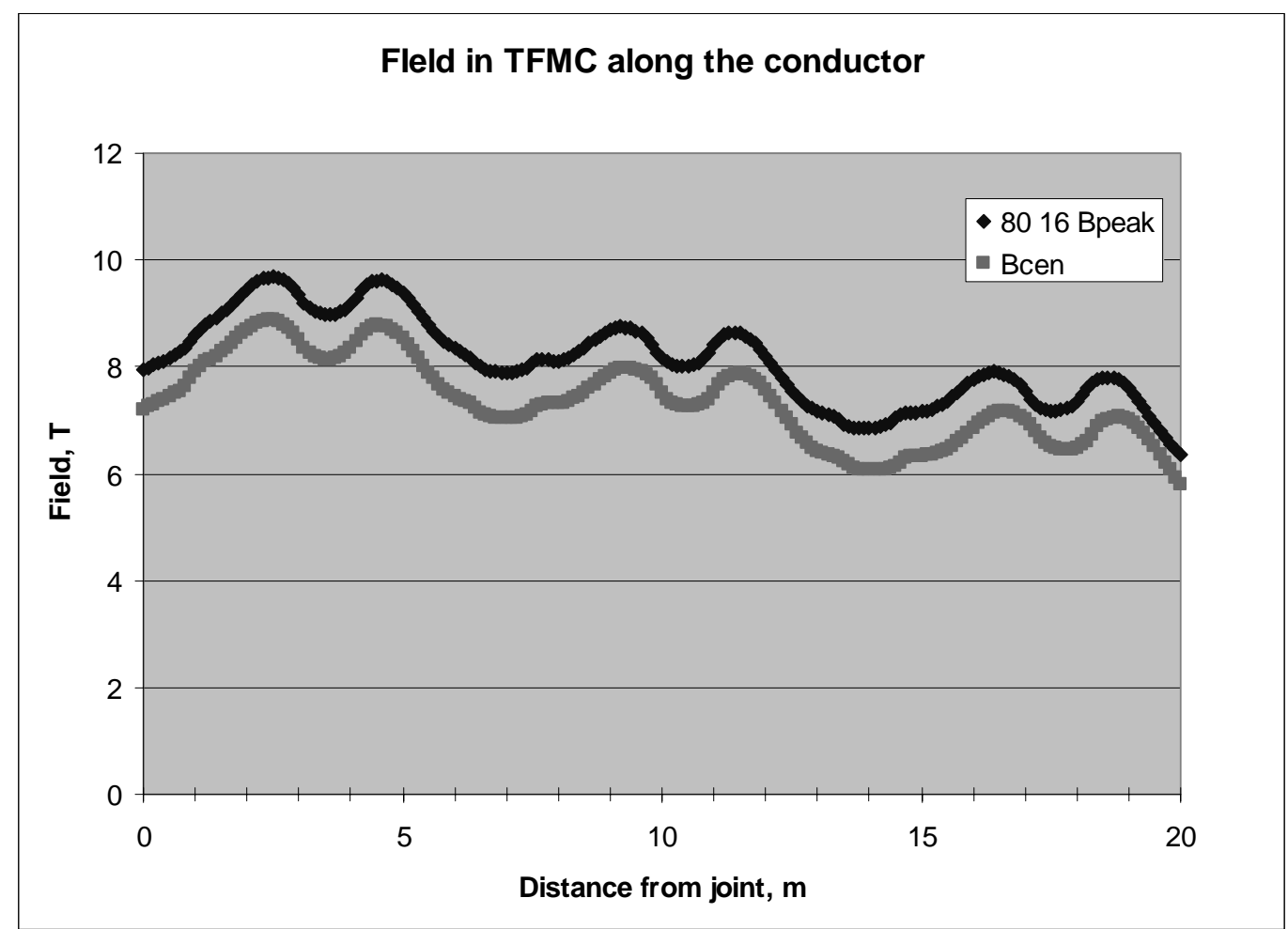

Fig. 1. Magnetic field distribution in the TFMC at $80 \mathrm{kA}$ in TFMC and $16 \mathrm{kA}$ in the LCT.

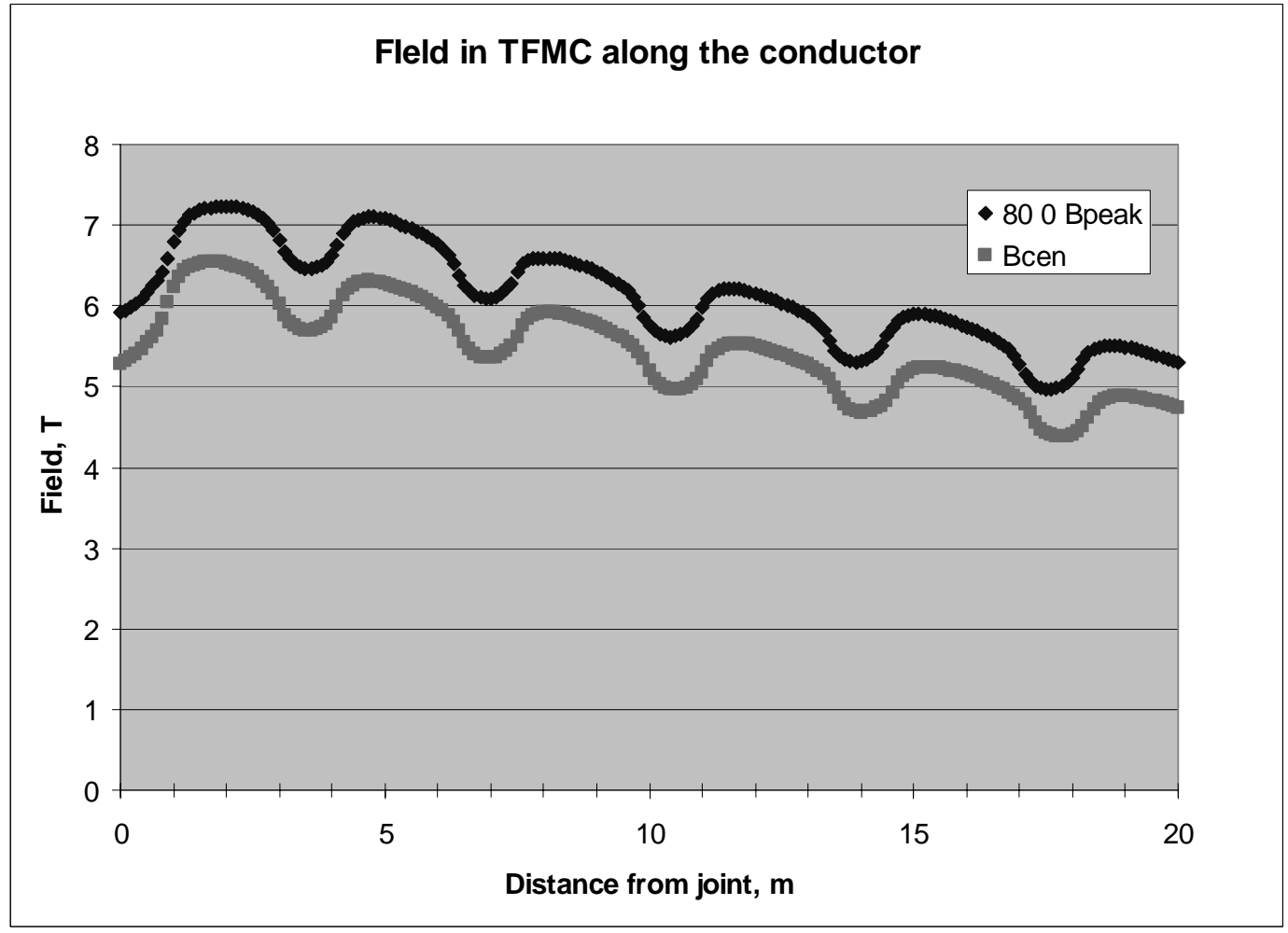

Fig. 2. Magnetic field distribution along the length of the TFMC conductor at $80 \mathrm{kA}$ in the TFMC and no current in the LCT coil. 
It is not readily clear what magnetic field represents the effective or equivalent magnetic field longitudinally. Therefore we need a realistic reconstruction of the electrical field and temperature profile. As it will be shown below we use in some cases Polito profiles generated by their M\&M code.

At the CS Insert analysis [2] the conclusion was, that the "effective" magnetic field is approximately equal to a median field between the peak field and the field on the center of the CICC [2].

The definition of the effective field corresponds to such a uniform magnetic field, when an average electrical field in the strands will be equal to the criteria of the critical electrical field, in most cases 10 $\mu \mathrm{V} / \mathrm{m}$.

In the CS Insert the ratio between the peak electrical field in the strand and an average electrical field in the strand is about $5: 1$, at $\mathrm{N}=20$ [2].

At the TFMC it is not so clear even how to compare the electrical fields due to the fact that the field along the conductor is not uniform and strictly speaking, there is a possibility that some strands never see as high field as the other strands, which go through the high field spots. This features of the TFMC require some analysis and also need a prospective views of how this nonuniform field affects comparison with the strand and how results of the TFMC performance will project onto ITER operation, where the uniform field along the straight leg of the TF magnet is expected on a length about $10 \mathrm{~m}$.

In this memo we will try to address the following questions:

How to extract the strand properties from the TFMC measurements and how it compares to the LMI strand?

What is the consequence of the nonuniform magnetic field distribution? Is it possible that some strands do not ever find themselves in the peak magnetic field and therefore can carry significantly higher current in comparison with those strands, which do enter the peak magnetic field? Then assumption of the uniform current distribution across the cross section may be replaced with a constant voltage between the joints along the strands and difference must be looked at.

How the TFMC test results are compared with the CS Insert and the TF Insert tests? What are the common features and differences?

\section{Uniform current distribution}

We start our study with the assumption of the uniform current distribution. Let us assume that across the cable the magnetic field is one-dimensional, in other words the field changes as a linear function of the distance, as shown in Fig.3.

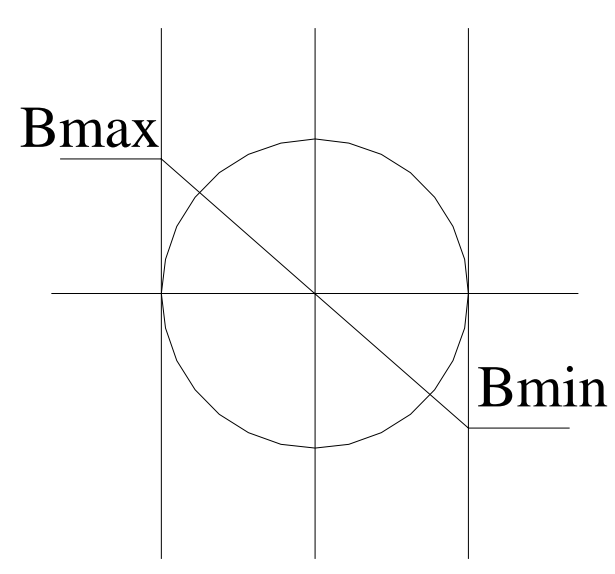

Fig.3. Distribution of the magnetic field in the cross section of the CICC cabling space (assumption).

When we have a uniform current distribution, naturally the electrical field in the cross section is nonuniform - higher where the magnetic field is higher and lower when the magnetic field is lower. 
For analysis we will use the following relation between the electrical field and magnetic field, current and temperature, a modified version of the relation proposed in [4].

$$
E=E_{c} \exp \left(\frac{I-I c\left(T_{s c}, B_{s c}\right)}{I o}+\frac{T-T_{s c}}{T o}+\frac{B-B_{s c}}{B o}\right)
$$

Here $\mathrm{Ec}=10 \mu \mathrm{V} / \mathrm{m}$, conventional "electrical critical field", the parameters Io, To, Bo are electrical field growth parameters. Change of a variable parameter, say temperature, by corresponding growth parameter To results in increase of the electrical field by a factor of $e=2.72$. The Io and Ic are related to a so-called N-

value as $\mathrm{N}=\mathrm{Ic} / \mathrm{I}$ o for the well known relation $E=E_{c}\left(\frac{I}{I c}\right)^{N}$

which is extended for non-isothermal and varying magnetic field conditions.

The Tsc and Bsc are current sharing temperatures at magnetic field at corresponding transport current Ic or in other words are parameters at which Ic(T,B) is defined at Ec, conventional "critical" electrical field, usually chosen at $10 \mu \mathrm{V} / \mathrm{m}$. We will use (1) since it is mathematically more convenient and has an explicit dependence versus T, B, Io, but within 2-3 orders of magnitude in electrical field variation the relationships (1) and (2) are almost equivalent, as was shown in [2]. To describe the state of superconductor we need a correlation between current sharing parameters, like Summers correlation [5] or University of Twente correlation by Godeke et al [6]. In our memo we will use the Summers correlation [5] with the CEA parameters defined later in the memo.

Relations between electrical field growth parameters Io, To and Bo could be found through corresponding derivatives [4].

$$
\left(\frac{\partial I}{\partial T}\right)_{E, B}=\frac{I_{o}}{T_{o}} \quad\left(\frac{\partial I}{\partial B}\right)_{E, T}=\frac{I_{o}}{B_{o}}
$$

Unfortunately, parameters To, Io and Bo are not constants but functions of the T,B, I and eps themselves. Therefore description of the $\mathrm{E}=\mathrm{E}(\mathrm{T}, \mathrm{B}, \mathrm{I}, \mathrm{eps})$ requires one more correlation - say $\mathrm{N}$-value, like $\mathrm{N}=\mathrm{N}(\mathrm{T}, \mathrm{B}, \mathrm{I}, \mathrm{eps})$. Until recently, there were no systematic attempts to come up with correlations on $\mathrm{N}$-value versus other variables. It was noticed [2] that practically all of the $\mathrm{NbSn}$ superconducting strands used in ITER project show reasonably good correlation in a form $\mathrm{N}=\mathrm{N}(\mathrm{Ic})$, regardless of the $\mathrm{T}$ and $\mathrm{B}$ (see for example [2], also presented at many ITER meetings). Since then, other researches confirmed that the $\mathrm{N}=\mathrm{N}(\mathrm{Ic})$ correlation is reasonably accurate (R. Maix, N.Kozlenkova, others). Although there is no fundamental understanding of why this correlation takes place, it is a useful observation, which allows comparing test results to strand properties in wide range of parameters.

Comparison of the $\mathrm{N}$-value measured on the CICC with the strand $\mathrm{N}$-value represents valuable information about CICC behavior and allow judging about transformation if any in the cable.

Until specified otherwise, we assume a uniform current distribution and will take a close look at the electrical field distribution in the conductor cross section.

Fig. 4 shows distribution of the electrical field in the cable cross-section at different $\mathrm{N}$-values.

It is seen that the peak value of the electrical field is significantly higher than the average field in the cross section. For $\mathrm{N}=20$, for example, it is almost 20 times higher than the average electrical field. This ratio is significantly higher than for the CS Insert due to higher current and therefore, higher self magnetic field. When current distributed uniformly, the peak electrical field significantly exceeds the average electrical field value, which we will address below in the memo. Since the run away in the strands starts locally, it means that the TFMC will have significantly lower value of the take off voltage than other CICC in more uniform field, such as the CS or the TF Inserts. 
Electrical field distribution in the cable cross section at different $N$ at average $E=10 \mathrm{uV} / \mathrm{m}$ ITFMC $=80 \mathrm{kA}$ ILCT $=16 \mathrm{kA}$

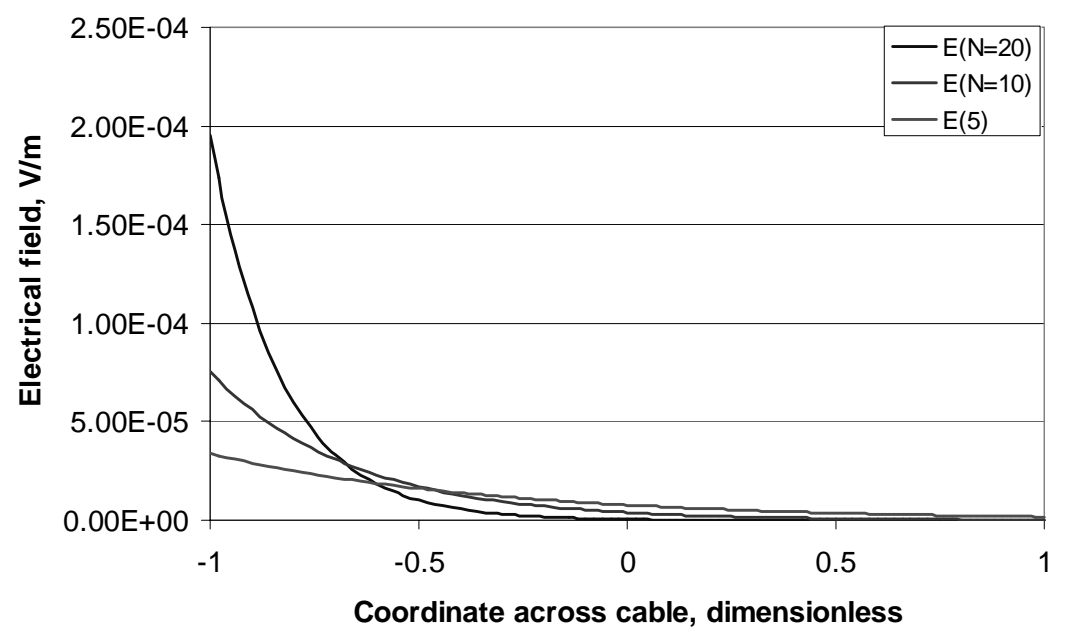

Fig. 4. Electrical field distribution in the cable with magnetic field distribution as shown in Fig 3. This particular case corresponds to ITFMC $=80 \mathrm{kA}$ and ILCT $=16 \mathrm{kA}$.

In the CS Insert and the TF Inserts the take off voltage exceeded 1-2 $\mathrm{mV}$, while in the TFMC it was 200$300 \mu \mathrm{V}$. In other words in the highly nonuniform electrical field distribution, the ran away of the magnet occurs at very low voltages, which makes the diagnostics very difficult or sometimes impossible due to the fact that the natural noise is not reliably exceeded by the useful signal. On the other hand, when $\mathrm{N}$-value is low, the ratio between the maximum electrical field and average electrical field is much smaller, thus a magnet with a low $\mathrm{N}$-value CICC is much easier to control and predict vicinity to take off current or temperature than a conductor with a high $\mathrm{N}$-value.

To compare strand performance to the CICC performance the most logical way is to use the strand characteristic and using the magnetic field and temperature distribution, figure out what will be the electrical field distribution and voltage between the joints, as measured in the experiment. However, such approach requires a computer code, like M\&M by Zanino-Savoldi Richard, for example, and lacks transparency of the "back of the envelope" calculations.

From the simplified stand point, let us try to answer the question: what effective magnetic field we should plug in into the Summers equation. For that we find a magnetic field, which would correspond to the "critical" average electrical field at given current. In earlier reports $[2,7]$ it was shown that for the CS Insert the effective magnetic field is about in between the average field and maximum field in the conductor cross section. For integration we use (1). Fig. 5 shows effective magnetic field for the TFMC for different $\mathrm{N}$-values, which shows that at $\mathrm{N}=20$, the equivalent magnetic field is in the middle between the average and the peak magnetic fields. However, at lower N, the equivalent field becomes close to the field at the axis of the cable. 


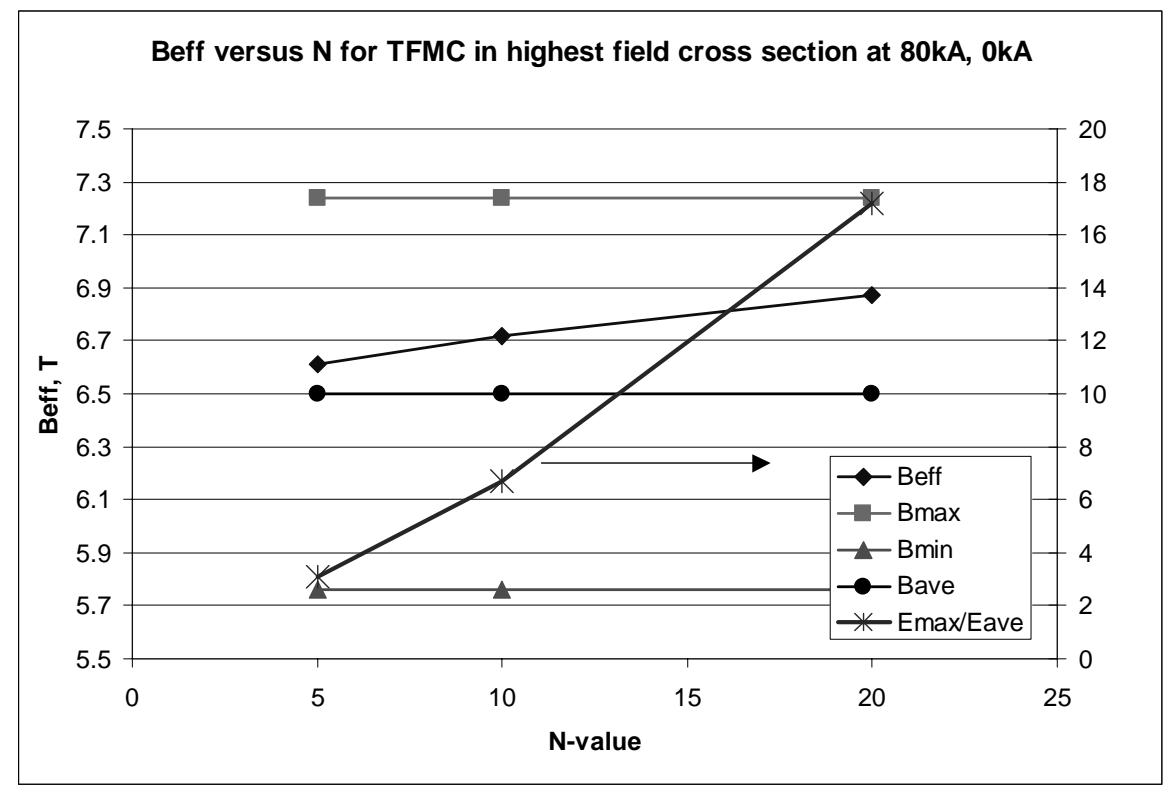

Fig. 5 Effective (equivalent) magnetic field for the TFMC cable at $80 \mathrm{kA}$ in TFMC. Peak to average electrical field is also shown

As it will be shown later, the $\mathrm{N}$-value at $80 \mathrm{kA}$ correspond to about 8-10 and a little lower for the shots with the lower transport currents. Therefore for our analysis we should take into account this dependence of the average electrical field versus $\mathrm{N}$.

Fig. 6 shows the equivalent field for 80kA and $16 \mathrm{kA}$ (TFMC and LCT, respectively) and it is seen that the tendency is about the same as it is for 80kA TFMC stand alone - at low N, the equivalent field is about average, at higher $\mathrm{N}$, the equivalent field is in between average and the peak field. Particularly, the effective magnetic field for the $80 \mathrm{kA}, 16 \mathrm{kA}$ in the TFMC and the LCT, respectively and at $\mathrm{N}=10$, the effective magnetic field is about $9.13 \mathrm{~T}$ at the peak field of $9.7 \mathrm{~T}$ and the median field of $8.8 \mathrm{~T}$. These values without this detailed analysis could have suggested that the "effective magnetic field" is about 9.25T, which would have been a slightly pessimistic estimate in field resulting to an overly optimistic estimate of the degradation.

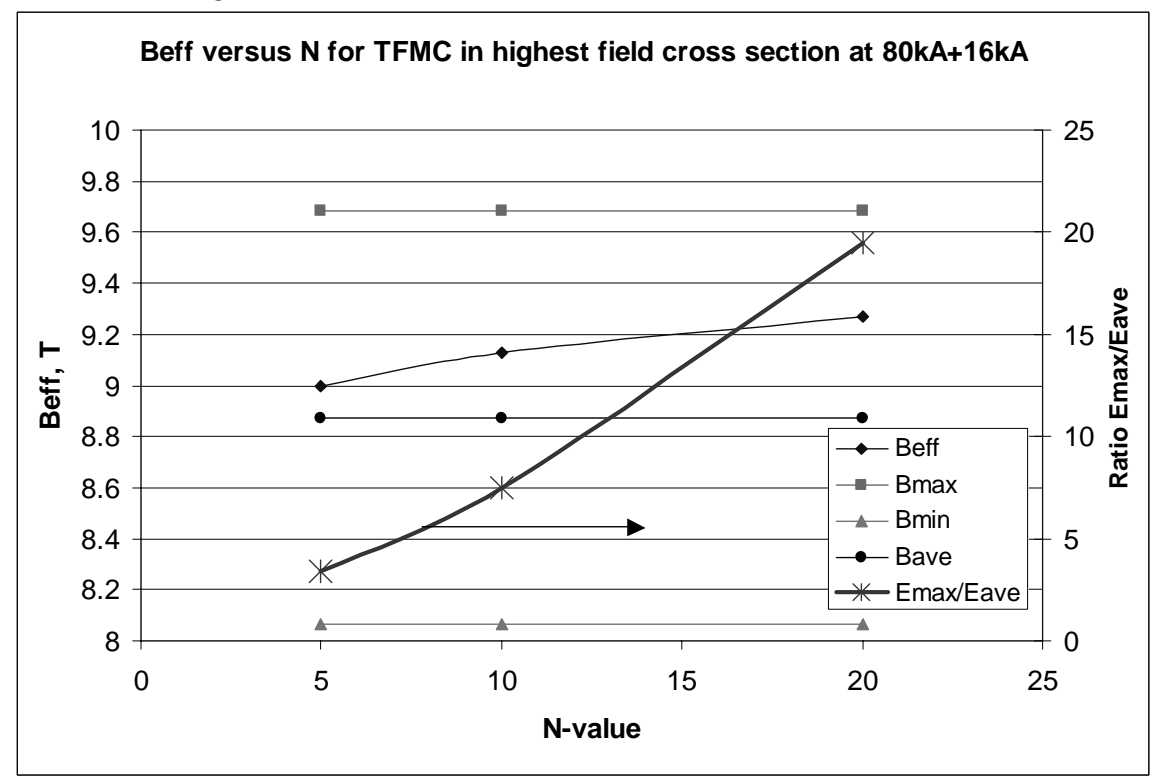

Fig. 6. Equivalent magnetic field for the CICC cross-section at 80kA in the TFMC and $16 \mathrm{kA}$ in the LCT coils. At $\mathrm{N}=10$, Beff=9.13T. 


\section{Analysis of experimental data on Tcs}

We will process the Tcs transitions using data taken by the voltage taps EK721 by the transient DAS and then use the corrected data from the sensor T712CX (inlet to the pancake DP1.2. The correction on the T712CX is required due to not exact gain in the amplifiers and a little bit shifted baseline. Walter Fietz was in the process of correcting the data by comparing the transient (or SPARTAN) data to the cyclic (slow) data acquisition sensor T712 and bringing the T712CX data in agreement with the cyclic data.

An example of such a correction by W. Fietz is shown in Fig. 7 for measurements of the Tcs at $45 \mathrm{kA}$ in TFMC, $13 \mathrm{kA}$ in LCT, which shows that the transient data temperature should be reduced by $0.2 \mathrm{~K}$ in this particular shot to represent the correct data for the region of my interest, where the electrical field is detectable. In some cases correction was not necessary, since the data in the transient DAS were either close enough to the cyclic DAS or already corrected by W. Fietz. W. Fietz by the way corrected the temperature valid for all range of temperature change, say from $4.2 \mathrm{~K}$ up to maximum temperature in any particular shot. I used sometimes a simpler correction to be close enough only in a narrow range of the Tcs determination.

Using this temperature data (usually filtered with $15-20$ points averaging) and smoothed EK712 data, I obtained typical plots as shown in Fig. 8 and Fig. 10-14.

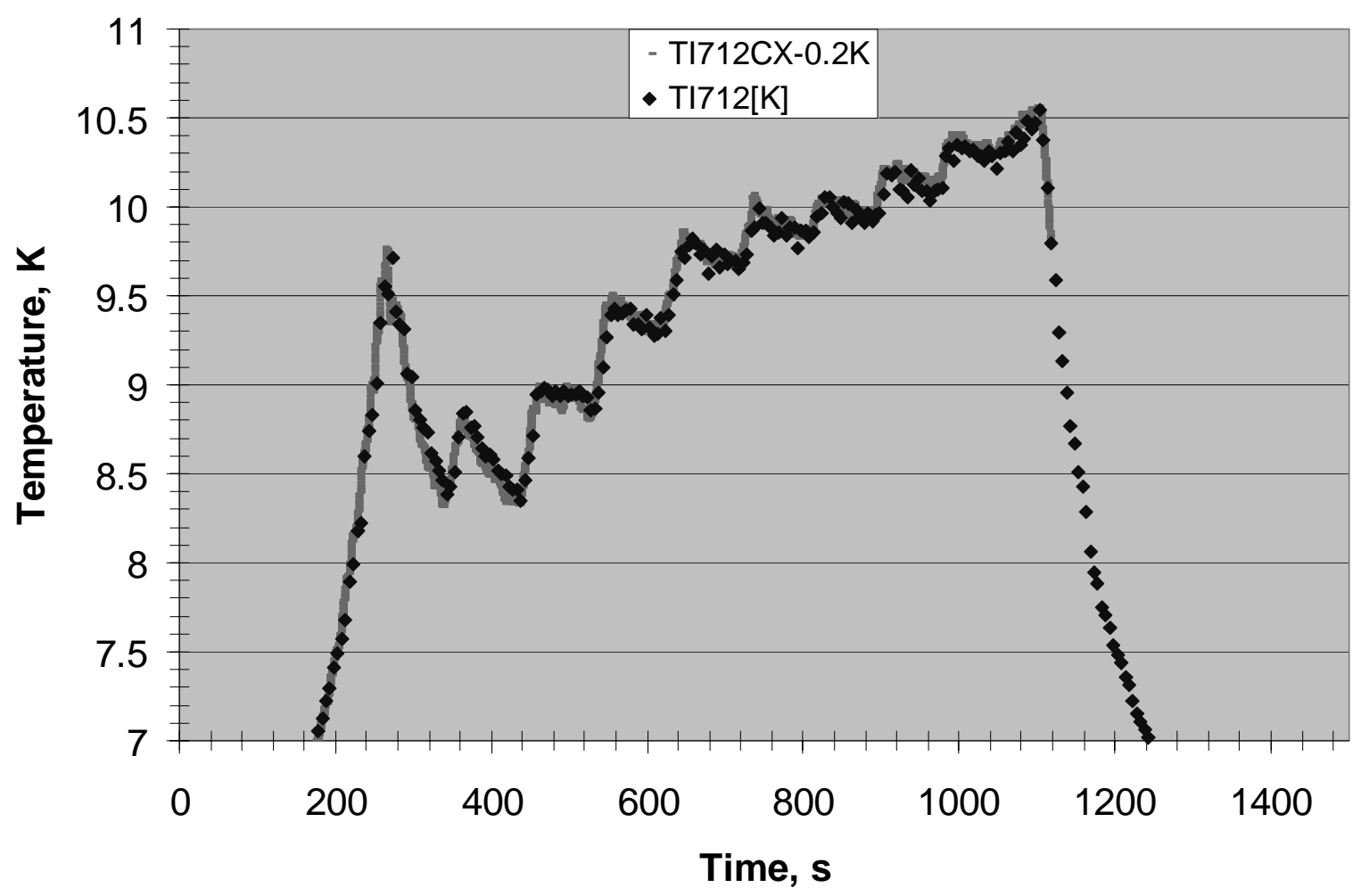

Fig.7. Temperature correction for the transient data to fit the cyclic data by W. Fietz.

Fig. 8 shows the voltage - temperature characteristic (VTC) and two fitting curves, which embrace the experimental points. Such a fitting is more or less judgmental, since number of points for smoothing is more or less arbitrary, but it seems that such a definition gives a flavor of the error bar. Also, strictly speaking for the $\mathrm{N}$-value determination the embracing curves do not necessarily represent the extremes, it is clear that through the cloud of the experimental points one can draw the VTC curves with higher or lower To. In this report we however assume that the embracing curves do represent the extremes and a correct value is somewhere in between.

We need to explain where from we are taking the level of voltage at which we define the current sharing temperature. This comes from integration by Torino team, distributed in the internal reports to the TFMC collaboration [8] shown in Fig. 9. It shows that at $25 \mu \mathrm{V}$ total voltage the electrical field in the strand 
reaches $10 \mu \mathrm{V} / \mathrm{m}$ averaged in the Bpeak cross section. Actually, Fig.9 is for $70 \mathrm{kA}$ in the TFMC, but at $80 \mathrm{kA}$ and $16 \mathrm{kA}$, TFMC and the LCT current respectively, the electrical field profile is assumed similar.

So, we choose $25 \mu \mathrm{V}$ total voltage as the Tcs criterion, which gives $10 \mu \mathrm{V} / \mathrm{m}$ average in the most "loaded" cross section of the cable. We can see that the test data fall in between To $=0.35 \mathrm{~K}$ and Tcs $=5.9 \mathrm{~K}$ and $\mathrm{To}=0.2 \mathrm{~K}$ and $6.3 \mathrm{~K}$. Using the CEA data for Summers equation for the LMI strand $(\mathrm{Bc} 2 \mathrm{om}=29.1 \mathrm{~T}$, $\mathrm{Tco}=16.9 \mathrm{~T}, \mathrm{C} 0=11000$ for $\mathrm{A} / \mathrm{mm} 2$ ) we can find that the derivative $\mathrm{dI} / \mathrm{dT}$ at eps=-0.7\% is about $32.86 \mathrm{~A} / \mathrm{K}$ (for such calculations we always operate with the current per strand, not total transport current) and therefore parameter Io is between $13.5 \mathrm{~A}$ and $9.9 \mathrm{~A}$, which at transport current of $111 \mathrm{~A}$ gives $\mathrm{N}$ value between 9.6 and 13.5. The range of the strains to match the data will be within $-0.64 \%$ to $-0.7 \%$. Therefore, average fit for the 80kA, 16kA (TFMC and LCT, respectively) corresponds to Tcs=6.1K, N=11, eps (operational) $=-0.684 \%$ and taking into account $0.094 \%$ strain from the EM load (Raff calculations given to me by Duchateau), the cooldown strain before charging is $-0.78 \%$, which is somewhat higher than expected from SS conduit subscale experiments by Specking and Duchateau, which showed compression of $-0.67 \%-0.7 \%$. The difference between $-0.67 \%$ and $-0.78 \%$ may look insignificant, but in terms of critical current at $12 \mathrm{~T}$ and $5 \mathrm{~K}$ it is more than $20 \%$ difference and it is a significant expense if that proportionally increases amount of superconducting strand, which need to be added to the conductor to reach the operating point with the desired margin.

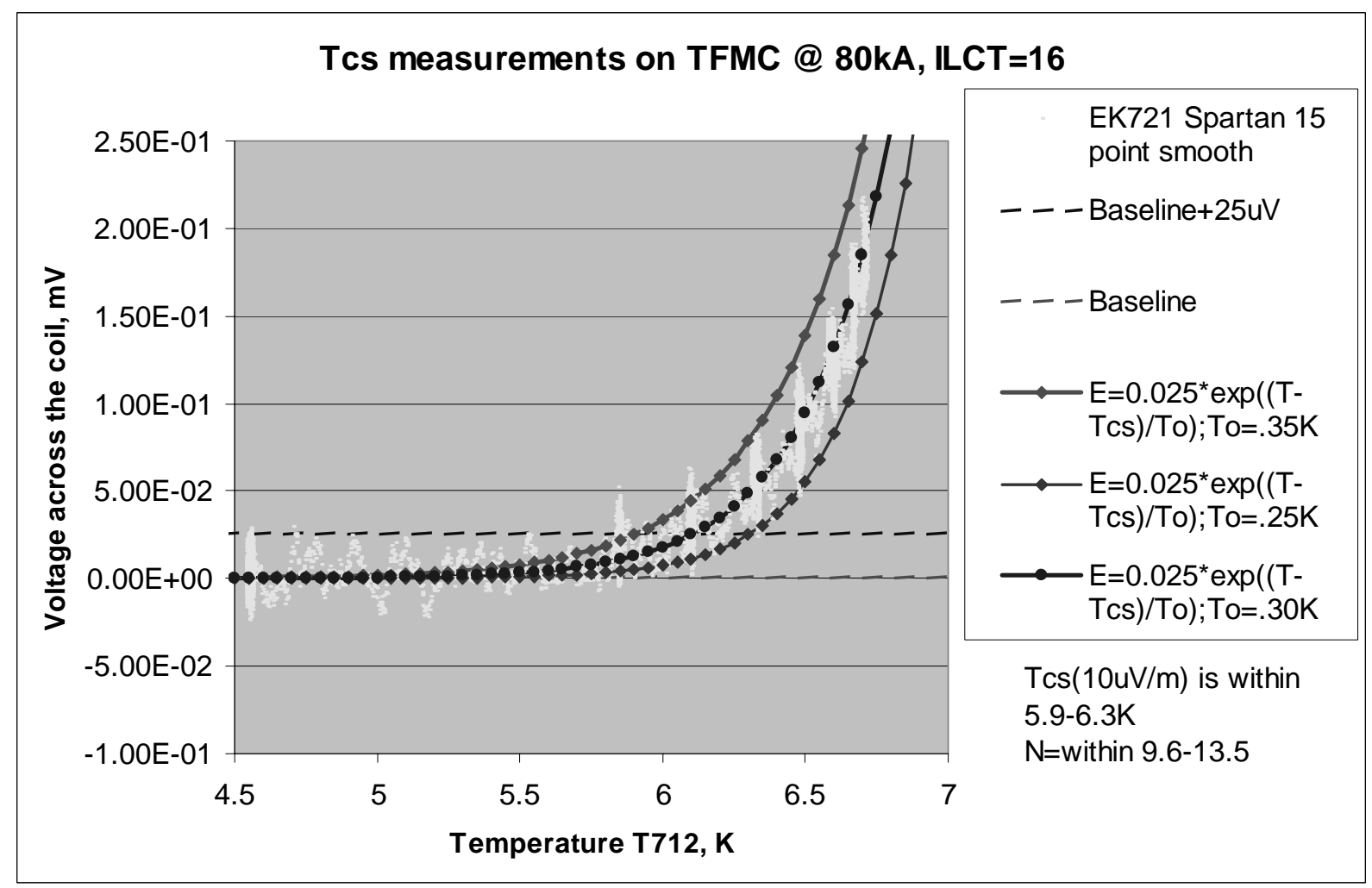

Fig.8. Volt-Temperature characteristic and fitting. 


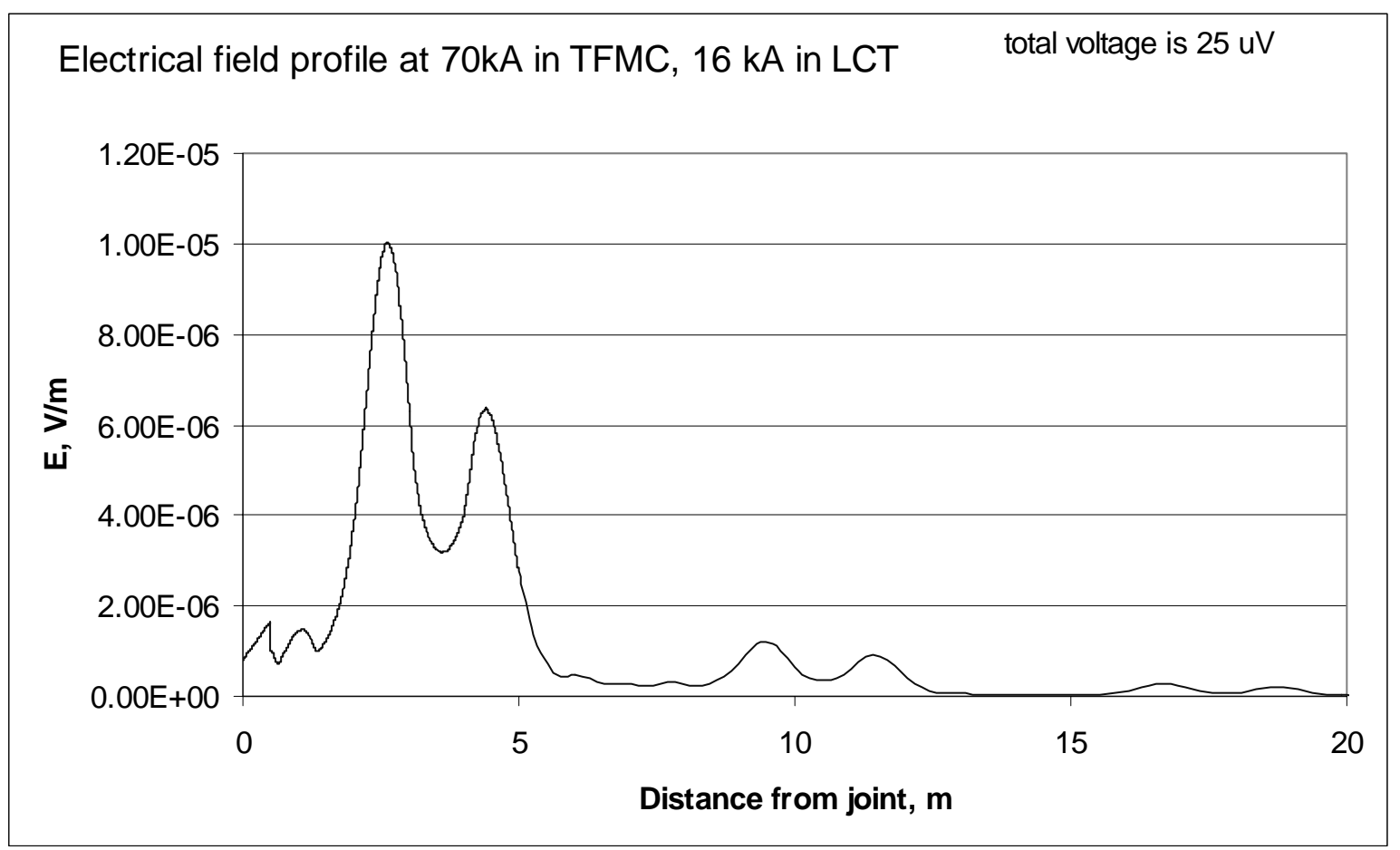

Fig. 9. Averaged electrical field profile along the length of the conductor (by R. Zanino et al, [8])

\section{Excurse into the alternative method of determination of the Tcs}

Before we proceed any further with the assessment of the Tcs, let us discuss in more detail how we define Tcs from the experimental VTC curve. Above we came up with an "equivalent" magnetic field which corresponds to the "critical" electrical field averaged across the cable cross section, assuming uniform current distribution. We also assumed by default that the voltage generating area is sitting in the longitudinally uniform field and that the length of the voltage-generating region is well defined. That is of course is not the case at all in practice. The contribution into the voltage from the area at the peak field is not overwhelming, the peak magnetic field (defined say from 98to $100 \%$ of Bpeak) is less than $1.5 \mathrm{~m}$, but the contribution into the electric field is noticeable over about 7-8 $\mathrm{m}$, according to [8]. Therefore there is not a lot of transparency about what equivalent magnetic field we should use if someone wants to avoid real field profile and substitute it with an equivalent magnetic field to compare with the strand. Intuitively, since in our previous studies with a longitudinally homogeneous field (CS Insert and other Inserts case) tells us that the effective field is somewhere in between the peak field and the field at the axis in this cross section, the TFMC conductor will have a lower effective field, since a lot of voltage comes from the conductor sitting in the lower magnetic field.

Let us consider an alternative approach. Theoretically if we had had all knowledge about electrical field distribution, the cleanest comparison of the strand in the cable to the strand stand-alone would be comparison of electrical field versus temperature at the peak magnetic field. Let us see what would be the difference in assessment of the Tcs and the effective strain if instead of using the average electrical field in the cross section we will use the criteria that a local electrical field becomes $10 \mu \mathrm{V} / \mathrm{m}$. In this case we should take the well-defined peak magnetic field, not an effective magnetic field. But for that case we need to know the local electrical field, which we do not measure. So we cannot avoid assumptions and we should find the total voltage across the pancake corresponding to the moment when the peak electrical field Epeak becomes equal to $10 \mu \mathrm{V} / \mathrm{m}$. Again we will use results in Fig.9 to calculate it.

Let us assume that $\mathrm{N}=10$ and try to estimate Tcs using local electrical field criteria instead of average electrical field. We can use results shown in Fig. 7 to see that when the peak electrical field is about 75 $\mu \mathrm{V} / \mathrm{m}$ when the average field is $10 \mu \mathrm{V} / \mathrm{m}$, which is 7.5 times higher. In other words, the peak field is 7.5 higher than the average electrical field. That means that the voltage at which we need to define the Tcs is 
7.5 times lower than $25 \mu \mathrm{V}$, or about $3 \mu \mathrm{V}$. That is a problem on its own - at $3 \mu \mathrm{V}$ the noise is so high that no direct assessment of Tcs possible. Therefore we have to extrapolate down to this level from the higher voltages, where the signal to noise ratio is more or less sensible. So, we use the relation (1) and try to find what temperature would correspond to $3 \mu \mathrm{V}$ of a "clean" voltage. Using To= $0.3 \mathrm{~K}$, and Tcs $(25 \mu \mathrm{V})=6.1 \mathrm{~K}$ we obtain that the correction to the Tcs for lower level of "critical" voltage is $0.3 \mathrm{~K} * \ln (7.5)=0.6 \mathrm{~K}$, thus $\mathrm{Tcs}($ Bpeak $)=6.1-0.6=5.5 \mathrm{~K}$. So, if we choose to use the local electrical field criteria and Bpeak, our parameters for determination of the prestress are $\mathrm{T} c s=5.5 \mathrm{~K}$, Bpeak $=9.68 \mathrm{~T}$, which gives eps $=-0.69 \%$, which is well within the error bar of $0.67-0.7 \%$ which we calculated above from the average electrical field and Polito criteria of $25 \mu \mathrm{V}$ for Tcs.

Thus both methods of calculation are approximately equivalent and unfortunately both require knowledge of $\mathrm{N}$-value and extrapolation into the low electrical voltage area where the noise is very high, higher than the signal. The method with the peak magnetic field and local electrical field requires extrapolation from the high voltage (tens and hundreds of $\mu \mathrm{V}$ ) into the lower level of several $\mu \mathrm{V}$, which is kind of uncomfortable, since the noise is high and certainty of extrapolation is shaky, but it eliminates the guesswork in finding the "effective magnetic field" for assessment. For both methods, knowledge of Nvalue is essential. At $\mathrm{N}=10$ or below, the "effective" magnetic field is much closer to the field on the axis than to the peak field, therefore we shall take this into account for comparison against the strand performance.

\section{Review of experimental data from Tcs measurements.}

Figures below illustrate Tcs measurements for most of the runs during the first and second TFMC campaigns. We will use for Tcs criteria the analysis by Polito group: at no current in LCT, the criteria is 40 $\mu \mathrm{V}$, at combined TFMC and LCT coil current we will use $25 \mu \mathrm{V}$. This is a simplification, for the cases when the LCT current is involved in other than $80 \mathrm{kA}$ or $70 \mathrm{kA}$ in the TFMC and $16 \mathrm{kA}$ in the LCT coil, but on the background of other uncertainties, this assumption seems reasonable.

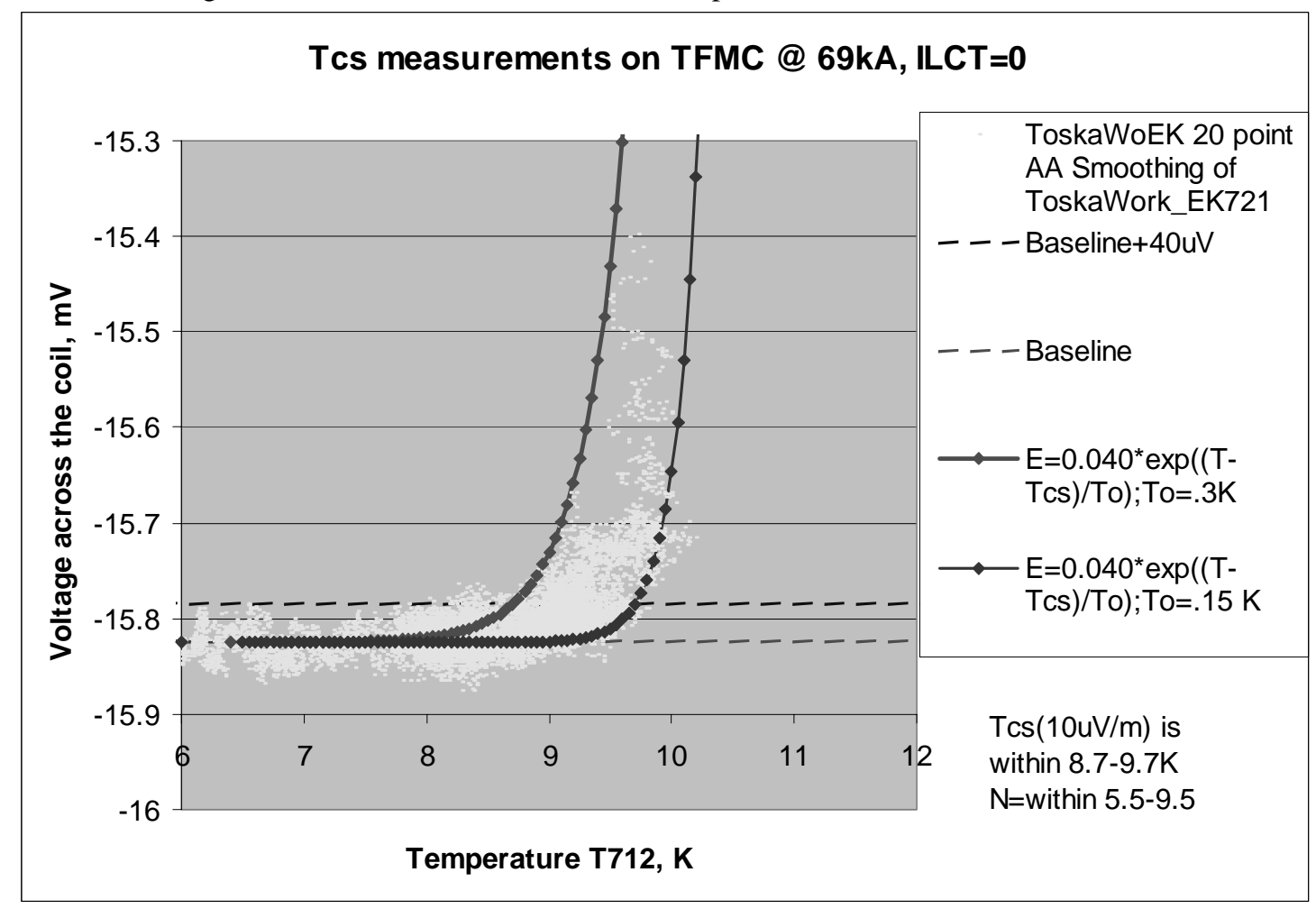

Fig. 10. Tcs measurements and fitting at TFMC current of 69kA 


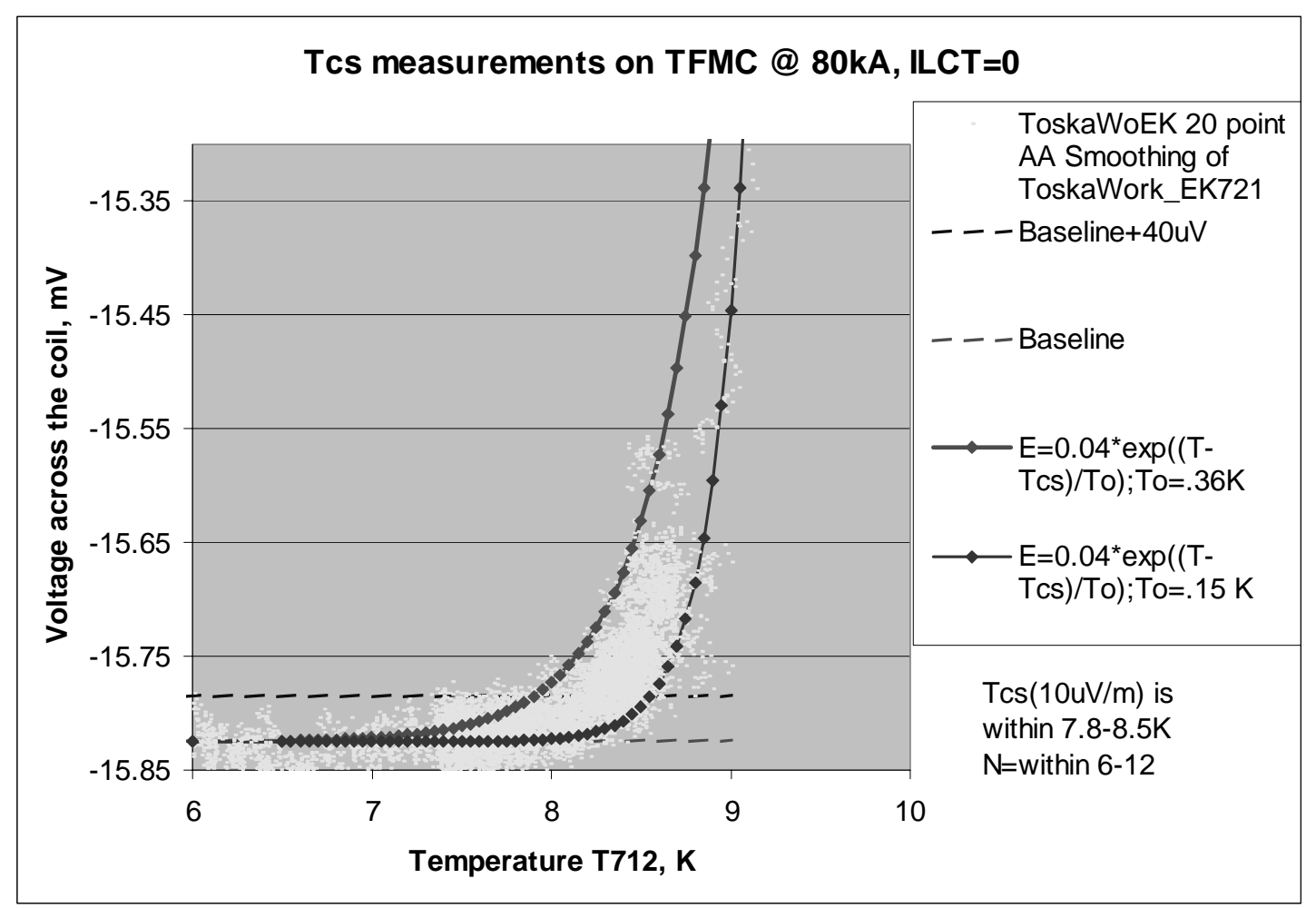

Fig. 11. Tcs measurements and fitting at TFMC current of $80 \mathrm{kA}$

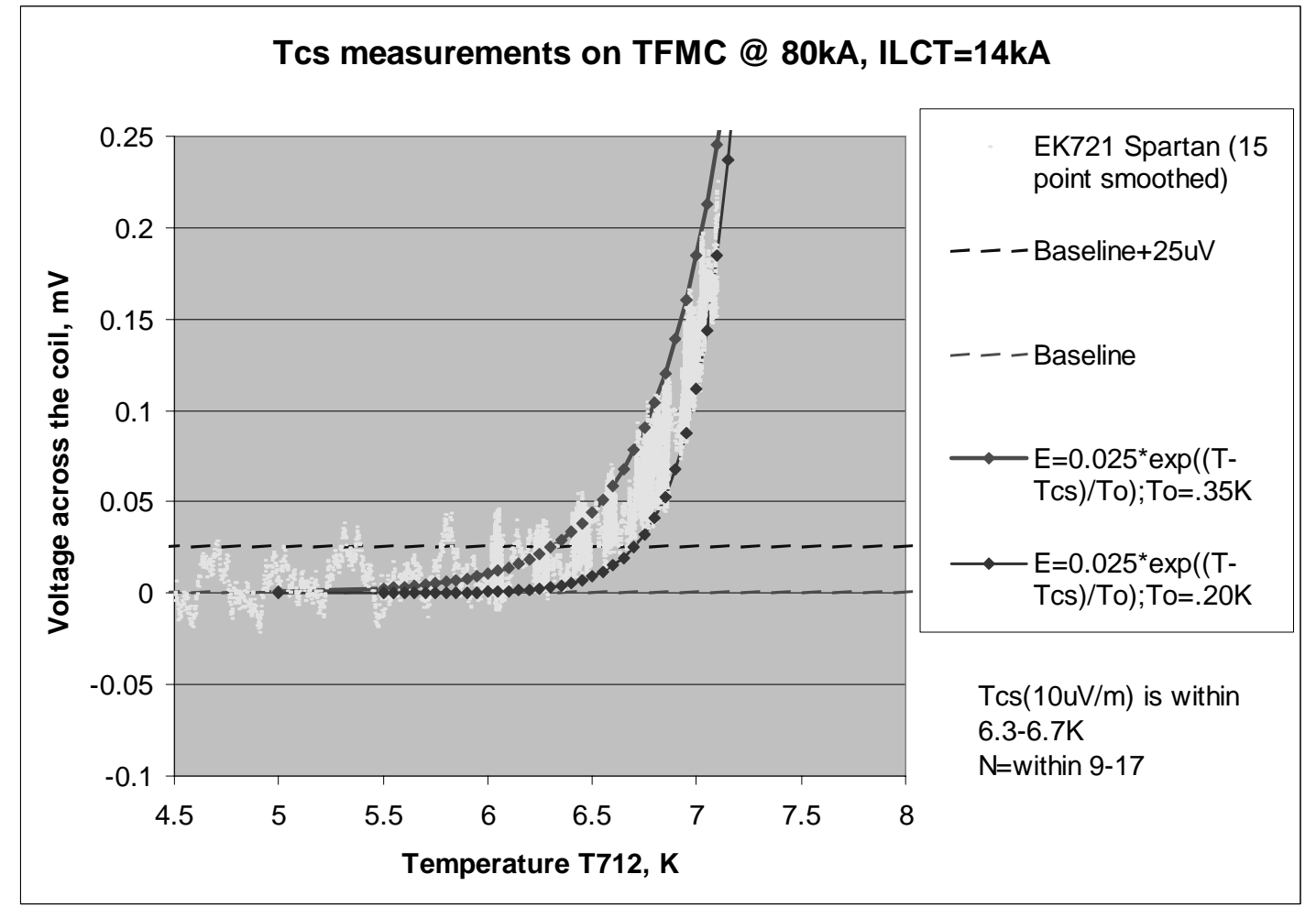

Fig. 12. Tcs measurements and fitting at TFMC current of $80 \mathrm{kA}$ and LCT current of $14 \mathrm{kA}$ 


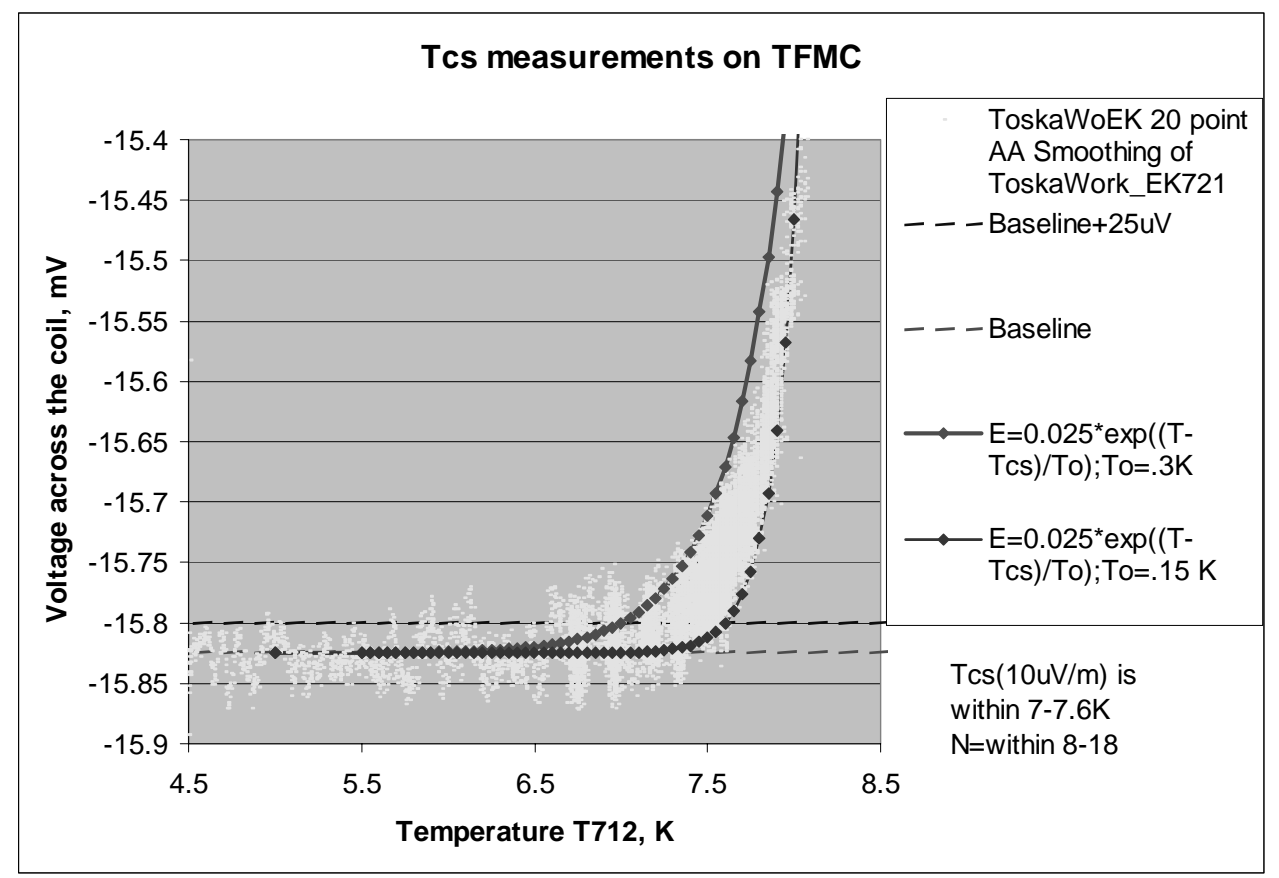

Fig. 13. Tcs measurements and fitting at TFMC current of $70 \mathrm{kA}$ and LCT current of $16 \mathrm{kA}$

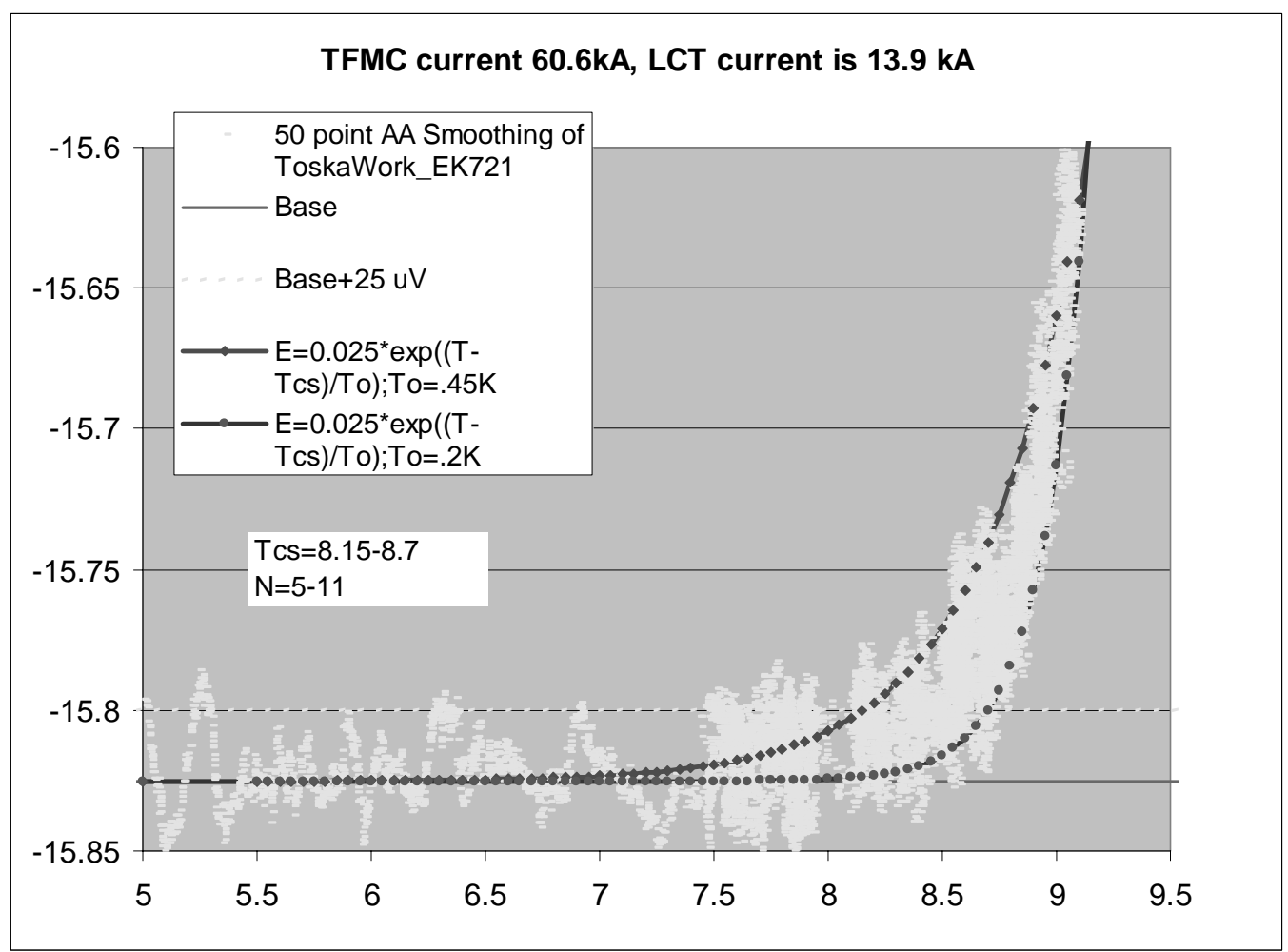

Fig. 14. Tcs measurements and fitting at TFMC current of $60.6 \mathrm{kA}$ and LCT current of $13.9 \mathrm{kA}$

All these data give us a range of the low and high bound of the Tcs. We fit the low and high bound data and find a fitting parameter - operating strain, which is expressed as:

$\varepsilon_{o p}=\varepsilon_{c d}+\varepsilon_{e m}$ 
which assumes that the operating strain is composed of the cooldown strain (which includes strain gained after the heat treatment and cooldown to RT, then all fabrication steps, then cooldown to operating temperature of $4.5 \mathrm{~K}$ ) and the electromagnetic (EM) strain comes on top of it.

To simulate the peak strain in the area of the peak field, which contributes the most into the voltage, we assume that the EM strain can be represented by the following relationship:

$$
\varepsilon_{E M}=k_{1} I_{L C T} I_{T F M C}+k_{2} I_{T F M C}{ }^{2}
$$

To find the coefficients $\mathrm{k} 1$ and $\mathrm{k} 2$ we use two points for EM strain, at 80kA, $16 \mathrm{kA}$ in the TFMC and LCT, respectively, the strain is $0.094 \%$, at $80 \mathrm{kA}$ and $0 \mathrm{kA}$, it is $0.08 \%$.

Thus for the Tcs test runs we find that the strains are as shown in Fig. 15.

Using this assumptions and after calculating the operating strains to fit the Tcs data, we obtain the fitting results for operating strain shown in Fig. 16.

An indirect credibility check on these results would be the same cool down strain in all runs. As we can see, with some scatter, the cooldown strain corresponds to $-0.75-0.8 \%$, which is higher than $-0.67-0.7 \%$ strain measured by Specking and Duchateau on stainless steel conduit samples [11].

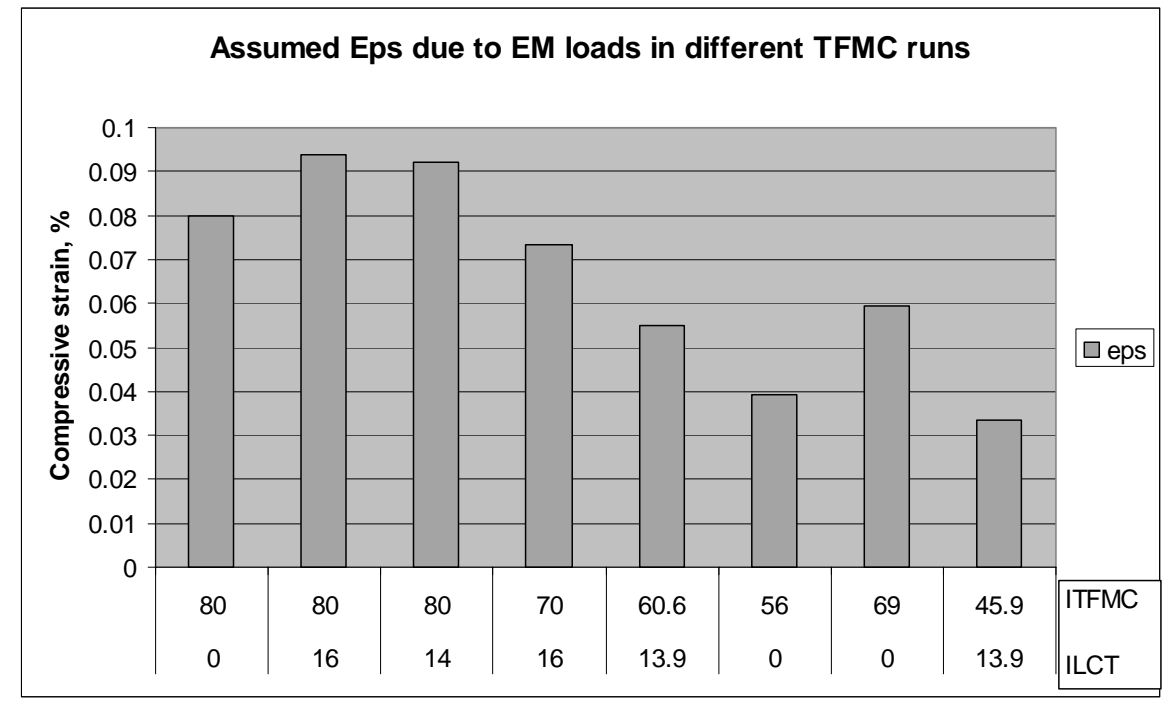

Fig. 15. Assumed EM strain in the current sharing area of TFMC

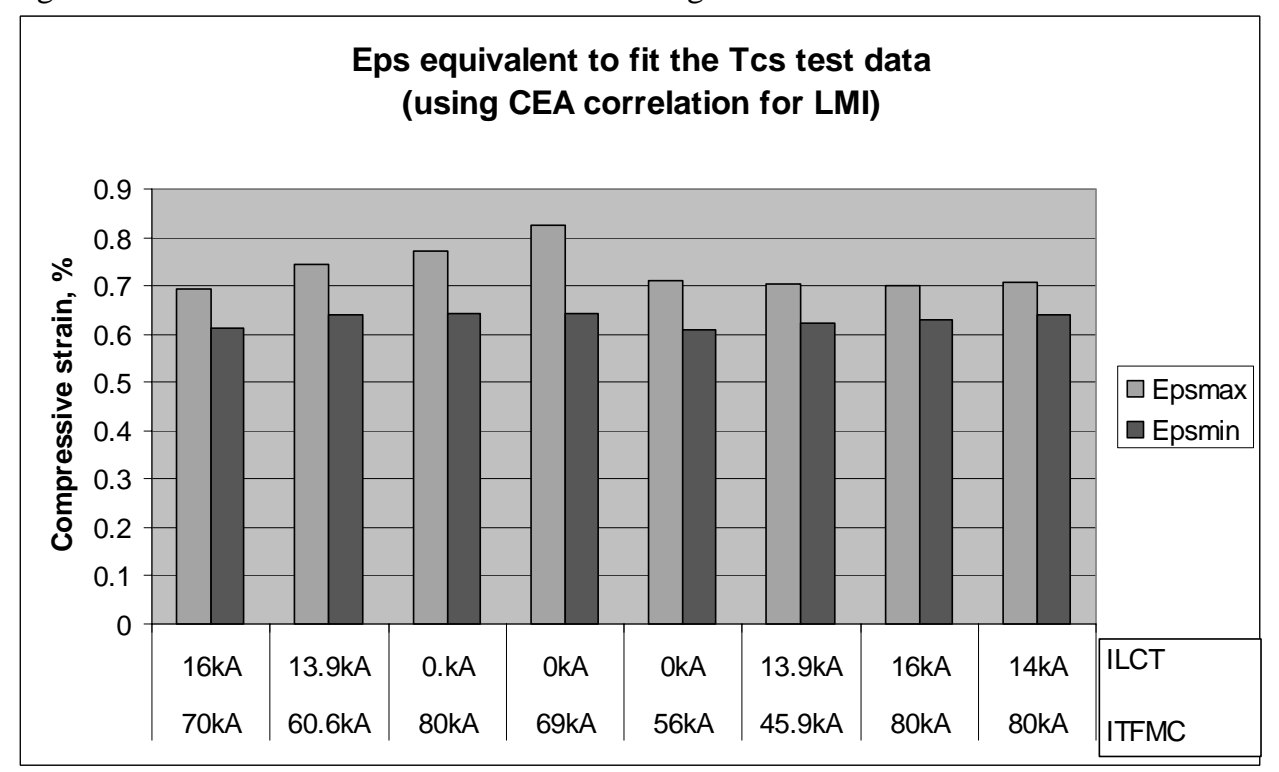

Fig.16 High and low bounds of operating strain in the TFMC runs 


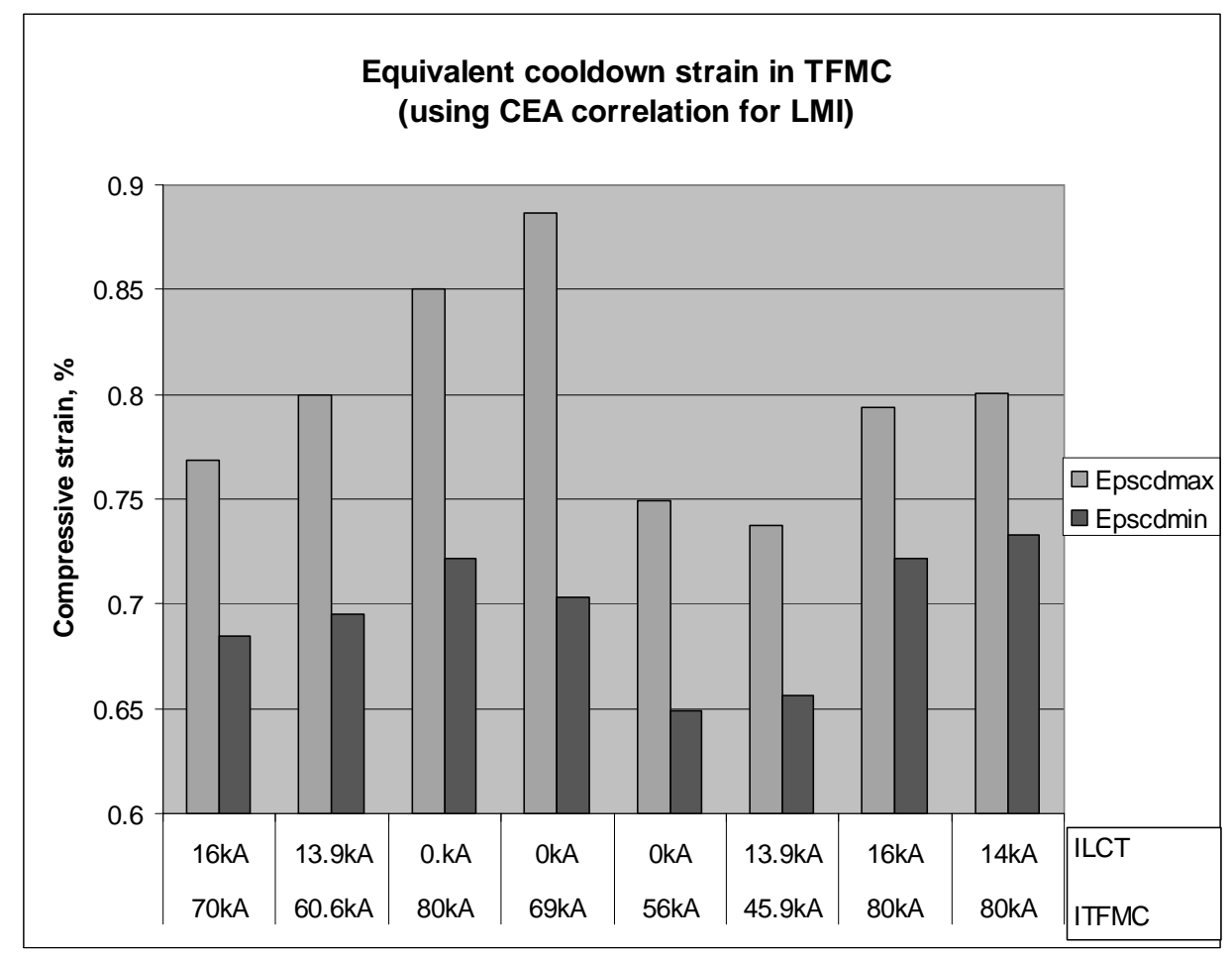

Fig. 17. Equivalent cooldown strain in the TFMC. High and low bounds.

\section{Effect of time delay between the temperature at the inlet and temperature at the current sharing area.}

Since the temperature measurements for the TFMC is judged by the temperature after the heater, there is a delay between the moment when the temperature is measured by the sensor after the heater and the moment when this temperature reaches the magnetic peak field, which results in the electrical field growth.

How essential is the delay? Since we have no sensors in between, we have to rely on code simulation. But in addition to the code we may have some flavor of what this time might be and what effect it can cause.

The minimum delay is the travel time between the temperature sensor and the place where the voltage is generated. Looking at the electrical field distribution (Fig. 9) we may say that this length is somewhere 3-5 $\mathrm{m}$ away from the joint and since the temperature sensor is some distance away from the joint (although between the sensor and the joint helium flows in a smooth tube) let us for order of magnitude analysis assume that this effective length is $6 \mathrm{~m}$. At 4 bar and $8 \mathrm{~g} / \mathrm{s}$ the helium velocity is about $0.2 \mathrm{~m} / \mathrm{s}$ and at $5 \mathrm{bar}$ it is $0.26 \mathrm{~m} / \mathrm{s}$, which takes 25 to $30 \mathrm{~s}$ for helium to reach the place of the voltage generation, although we should take into account that it is hard to be accurate there because at a high heat load, there are some oscillations taking place, which make a noise.

But to form a more or less steady temperature profile and corresponding voltage, it takes a while. In our experience on the PTF, where the temperature sensors were placed in the central channel of the CS conductor, formation of the temperature profile took significantly longer than just a travel time. Fig. 18 shows the formation of the temperature profiles in the PTF tests before and after the joint. As one can see, the response time between beginning of the temperature growth on sensors before the joint is about $10 \mathrm{~s}$, while reaching the temperature flat top time difference is significantly longer - about 30 seconds. The reason for this delay is the heat exchange between the helium and the CICC, which takes some time to come to equilibrium. 


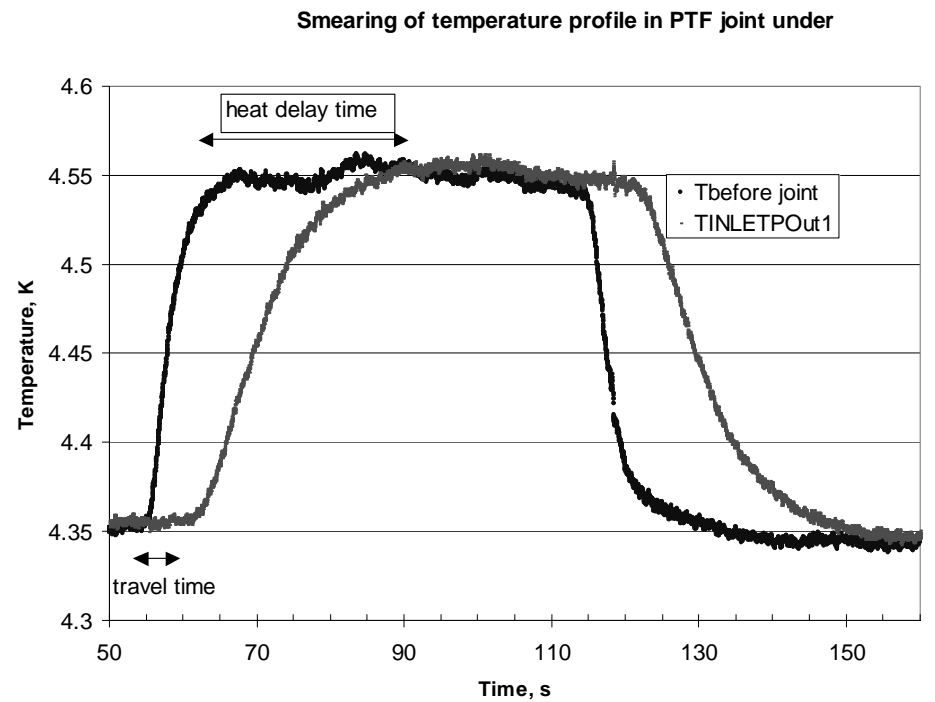

Fig. 18. Time of the temperature profile establishment is tens of seconds longer than helium travel time in the CICC and joint

Unfortunately, there is no direct temperature measurement in the TFMC to judge about how long it takes to get to the equilibrium and what level of temperature is in the voltage generating area, but we can indirectly get a feel about it comparing the data from the voltage taps and the inlet temperature sensor.

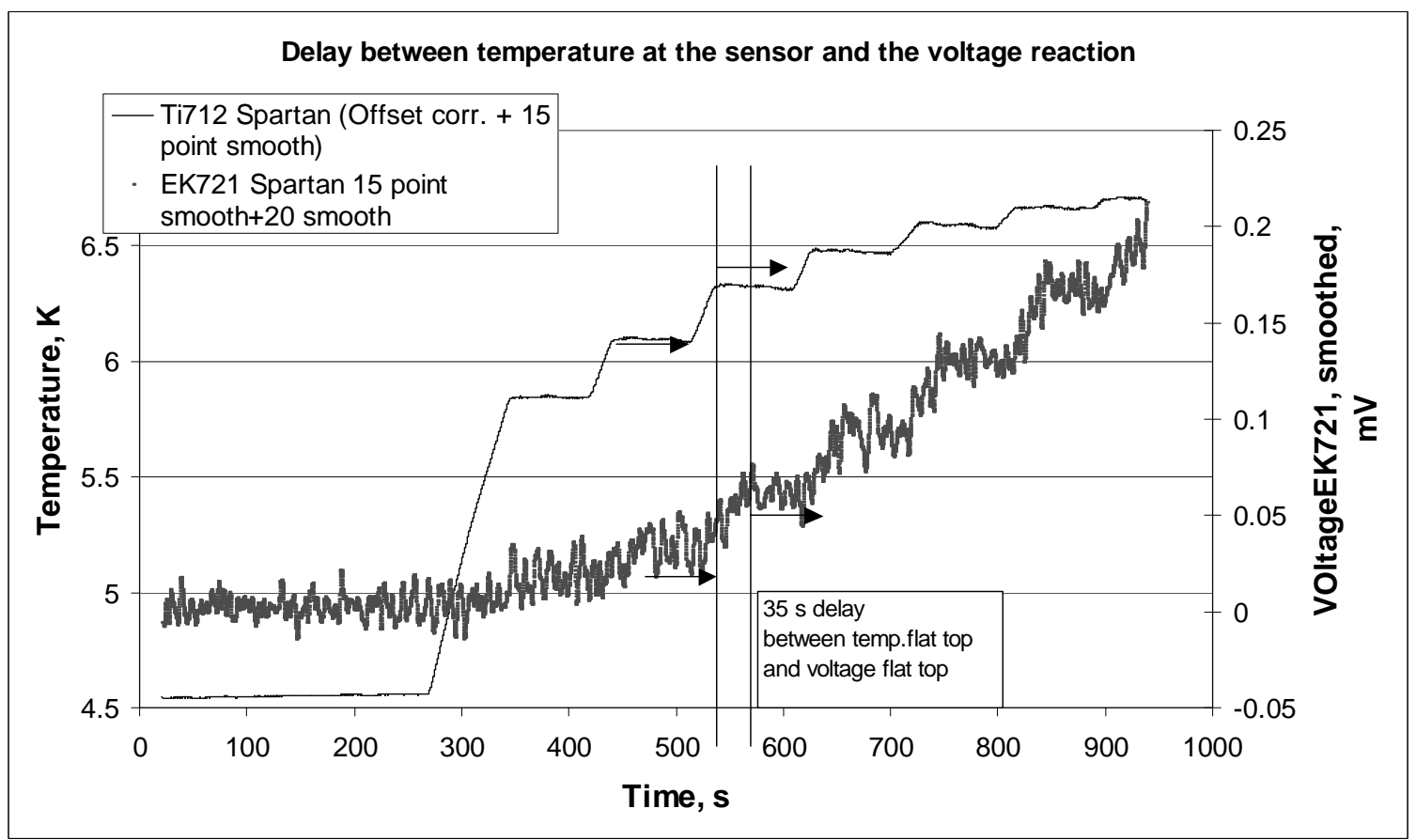

Fig.19. On the delay time between inlet sensor reading and the voltage across the TFMC pancake 1.2

Fig. 19. shows the Tcs measurements: temperature after the heater and voltage in the coil are shown. If one tries to synchronize when the temperature stabilizes after the power incremental increase on the heater and when the voltage is stabilized, one can see that the difference in this run is about $30-35 \mathrm{~s}$, as shown in Fig.19. 
Fig.20 compares two VTC transitions for this Tcs run. First, when the voltage and temperature are taken in the assumption that there is no delay between the temperature growth and the voltage growth. The second VTC is for the case when we synchronize the voltage in the temperature by shifting the time scale by $30 \mathrm{~s}$ back to allow the warm helium to reach the equilibrium in the voltage generating area. Strictly speaking, instead of continuous curve voltage - temperature we should pick only "equilibrium" points and that would constitute the real VTC. My attempts to do that yielded a curve close to the curve with a delay shown in Fig.20.

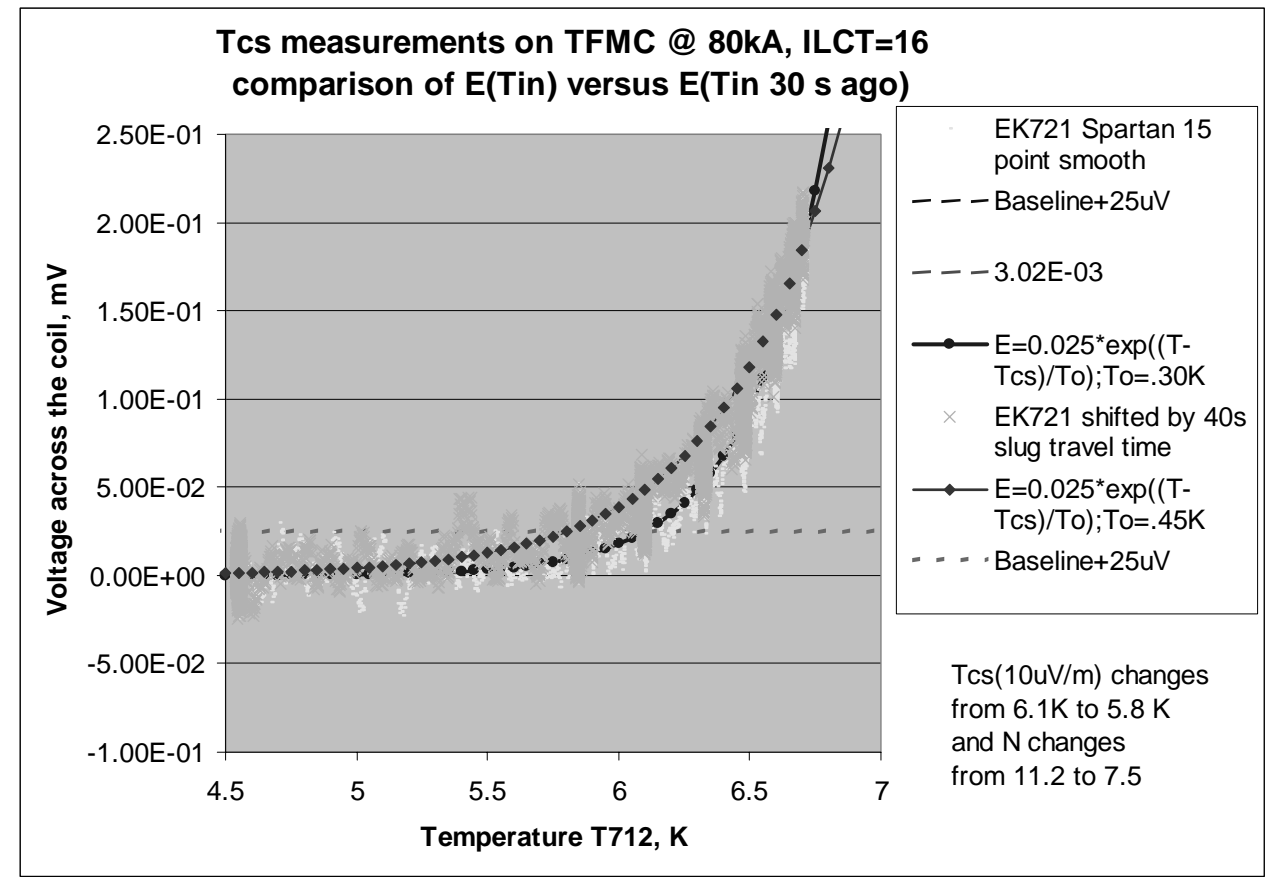

Fig. 20. Comparison of the VTC with and without correction for time delay between the emperature sensor signal and voltage signal across the DP1.2 pancake

As one can see from the plot, this correction shifts Tcs lower by noticeable amount (from $6.1 \mathrm{~K}$ to $5.8 \mathrm{~K}$ ) and that the $\mathrm{N}$-value becomes lower (from 11.2 to 7.5 ). We still assume that the temperature in the heat generating area is the same as after the heater.

This correction would have shifted the fitting parameter of the operating strain from $-0.67-0.7 \%$ to $0.73-0.76 \%$ (at $\mathrm{N}=10$ ) and the cooldown strain into the area below $-0.82 \%$ which is not insignificant result and implies that the degradation is even worse than we thought before.

This phenomenon deserves a more careful study and should be taken into account for processing the test data. In this memo we do not account for that, but should keep in mind that the real degradation is probably worse than what we see from $\mathrm{E}$ (Tinlet) curves without delay effect.

\section{$\mathrm{N}$-value measurements}

We processed all VTC measurements carried out on the TFMC and calculated Io and $\mathrm{N}$ for high and low bounds, using relationships (1-3) and the correlation for Ic(eps,T,B) given by CEA at corresponding eps, which fit measurements. Determining the $\mathrm{N}$-value we did not make any corrections on the time delay, discussed above, therefore our estimates of the $\mathrm{N}$-value are probably on the high side. As we can see from the VTC curves, the N-value definition is not very accurate, but hopefully, we still can see some tendency in the data.

Fig. 21 shows the results of such calculations. 


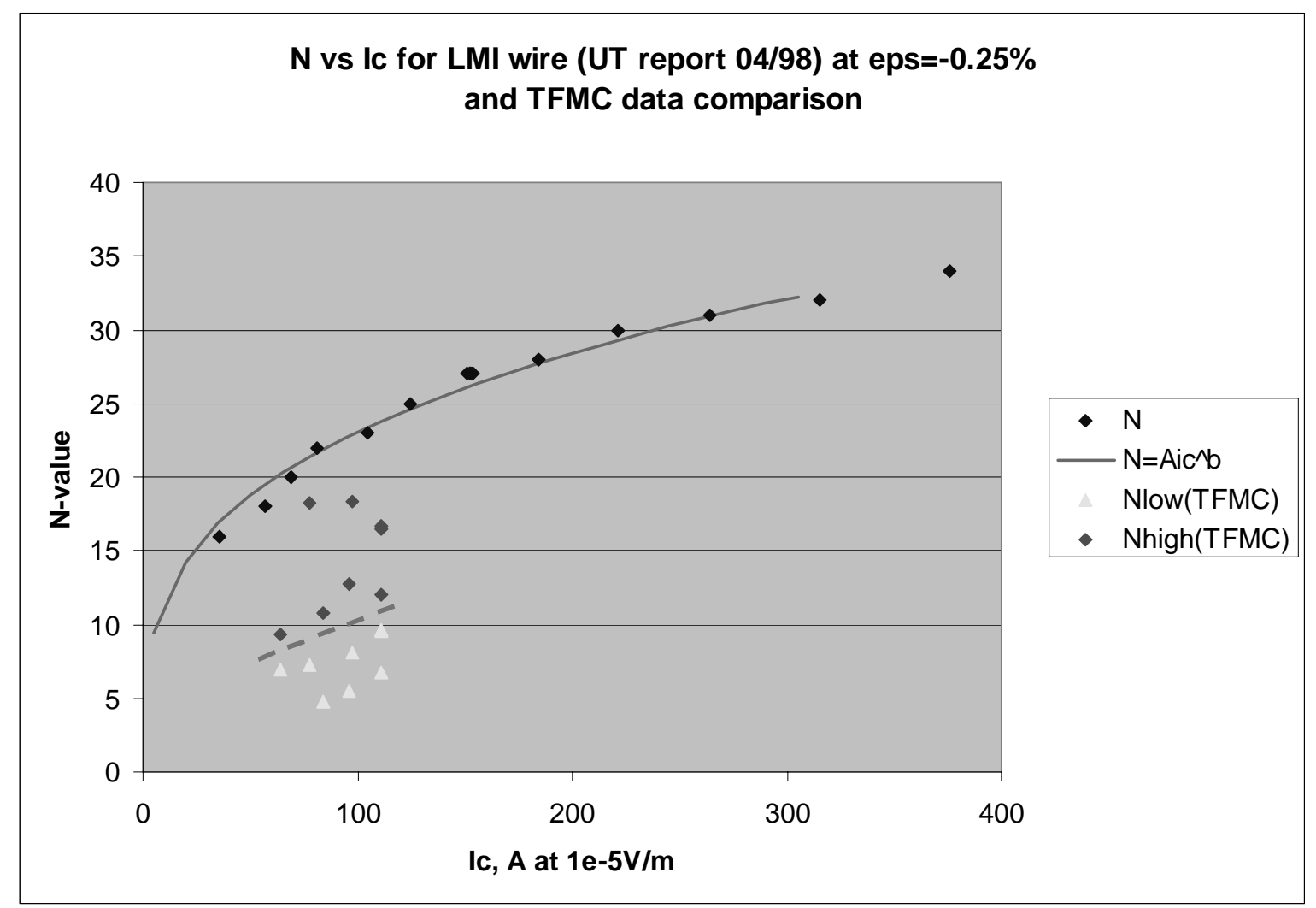

Fig. 21. N-value deduced from the TFMC testing and comparison with the $\mathrm{N}$-value measured on the LMI strand (University of Twente data processed by N.Martovetsky)

One of the purposes of the $\mathrm{N}$-value measurements is to determine if the strand properties have some change, which would indicate if the cable experienced any degradation in addition to anticipated compression by stainless steel conduit.

Unfortunately, we do not have any data on the LMI strand for N-value versus strain to know how sensitive it is to strain. The data available on the FURUKAWA, shown in Fig. 22 [2] suggest that N-value may noticeably drop under strain, especially between strain free condition and the tensile strain. However, the difference between $-0.24 \%$ and $-0.6 \%$ suggest that this change is not as dramatic as we see in Fig. 21 , where $\mathrm{N}$-value dropped down by a factor of 2 . Thus, it seems that the strain of $-0.7 \%$ does not completely explain the $\mathrm{N}$-value reduction, which may suggest that there may be some additional mechanism which transforms the strand properties between a free strand and a strand in the CICC, similar to those observed in the TF Insert and the CS Insert, where reduction in N-value was associated with degradation of Ic by 25$40 \%$. In this respect the effective cooldown compressive strain in the TFMC of $0.75-0.8 \%$ may be interpreted as expected compression of $-0.6 \%$ and some additional degradation. Such interpretation is indirectly supported by the observation that measured Tc (0T) at 16.2-16.5 $\mathrm{K}$ is not suggesting such a high strain as $-0.75-0.8 \%$, but this conclusion is based only on the CEA parameters for Summers equation which was never studied in the Tc (0T) area. Unfortunately, strand experimental data about Tc at $0 \mathrm{~T}$ under stress are unavailable and therefore the question if the TFMC conductor behavior could be fully explained by expected compression of steel conduit remains open and require more study.

\section{How non-uniform current distribution can be in the cable due to a small magnetic field spot in the TFMC?}

Our analysis so far was based on the assumption that the distribution of current across the cross section of the CICC is uniform. 


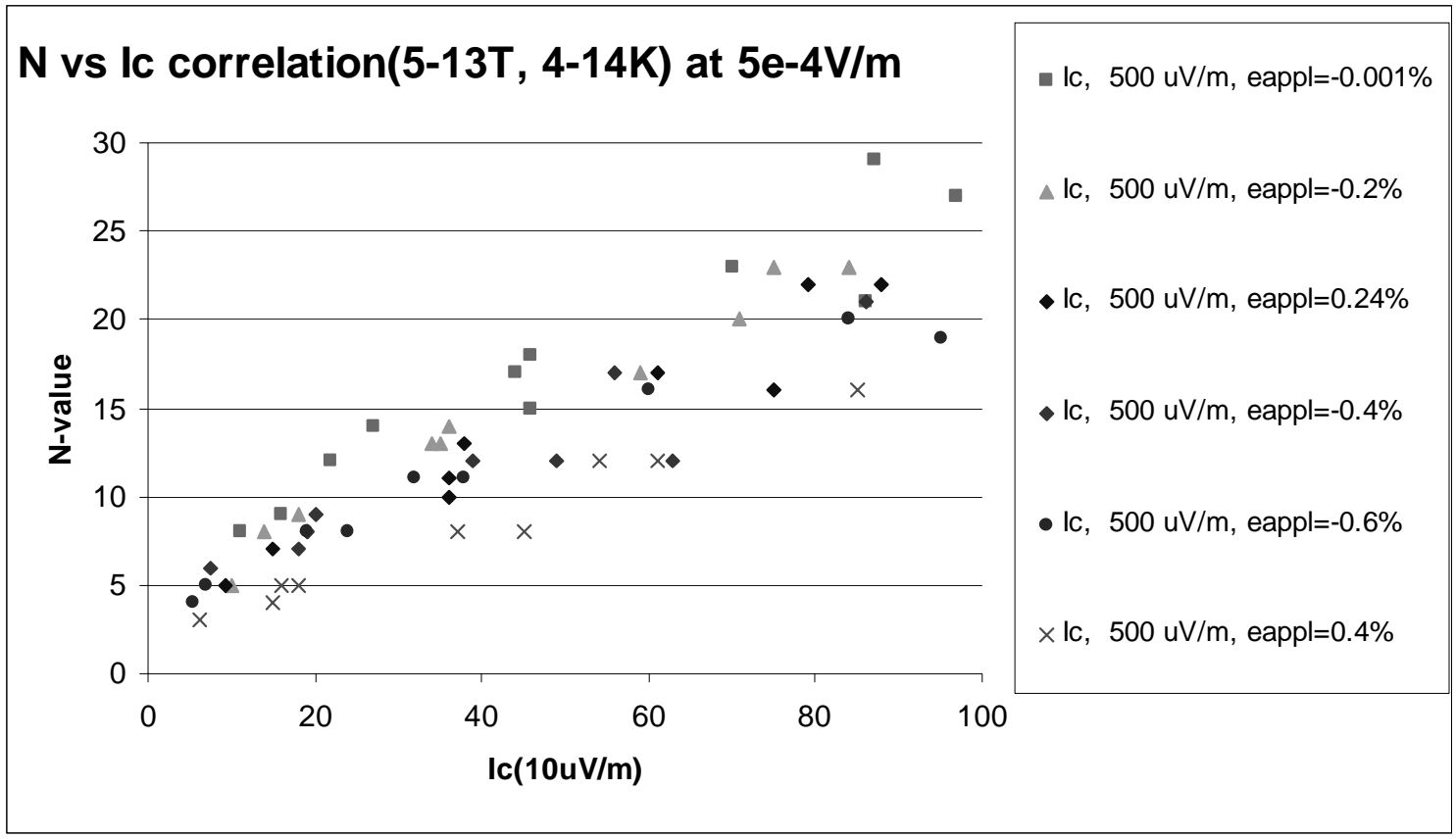

Fig.22. $\mathrm{N}$ value vs Ic correlation for selected data (see text for explanations)

Two previous studies [2] and [7] showed that the credible resistances in the cable do not provide sufficiently low resistance to transfer the current between the strands on a length of half twist pitch of the longest cabling twist pitch of about $450 \mathrm{~mm}$ at electrical fields of several $\mu \mathrm{V} / \mathrm{m}$.

That means that the current will distribute in the strands according to their integrated voltages along the length, not their local electrical fields and the ease of distribution depends primarily on the quality of the joints and interstrand resistances in the joints and to a lower extent it depends on the length of the cable between the voltage generating area and the joints and interstrand resistances in the cable.

A nonuniform current distribution due to the joint imperfections always exists in the CICC at some level due to different resistances between the strands to the current terminal. However, when a significant voltage is developed along the strand during the Tcs measurements it equalizes current distribution in the cable. So the question is if in our tests we had enough voltage developed along the length in the TFMC. This phenomenon, as it was discussed in many studies, may potentially show a performance on a model coil worse than in a large ITER magnet, since in a large ITER magnet the voltage which develops along a longer length will effectively suppress the imperfections in the joint more efficiently.

Unfortunately, there is no direct instrumentation in the TFMC to prove or disprove this effect. However the likelihood of the nonuniform current distribution in the CS Insert is thought to be very low, since we had a long length of a conductor sitting in a uniform (longitudinally) magnetic field and total voltage between the terminals reached in some cases $2 \mathrm{mV}$, which did not affect the VTC shape. This voltage is more than sufficient to suppress the non-uniformities in the joint resistances. We do not know the scatter in the joint resistances, but we assume that the voltages between the strands in the joint inside the cable are smaller than the voltage between the terminals. In the CS Insert, the joints were on the order of $2 \mathrm{nOhm}$, which gives at $40 \mathrm{kA}$ about $80 \mu \mathrm{V}$, which is 25 times smaller than the longitudinal voltage. Strictly speaking I am not aware about any direct crucial test on that matter, but indirectly we know that the joint resistance does not grow too much or at all as the transport current goes up until quench current, which suggests that all strands participate in carrying the current. In the CSMC Inserts we could observe the VTC in a relatively wide range to see if $\mathrm{N}$-value is about the same at several micro Volts per meter as at several tens of micro Volts per meter. In the TFMC the range of voltage, which would be not very noisy is very limited, so it is impossible to get reasonable evidence about if VTC of the TFMC is distorted by the nonuniform current distribution.

At the level of voltages of 100-200 micro Volts, the level of voltage is sufficient to suppress the joint nonuniformity (joint voltage at $1 \mathrm{nOhm}$ and $80 \mathrm{kA}$ is $80 \mu \mathrm{V}$ ), but there is no guarantee that it is the case 
with the TFMC. So our analysis in this memo is based on the assumption that the joints do not affect the current distribution, but it is not possible to produce a crucial proof from the test data if they do or do not.

In this section we will explore is the effect of a very localized magnetic field and thus electrical field in the TFMC and see if non uniform current distribution is possible not due to joints irregularities, but due to different exposure of the strands in the cable to the magnetic field. We will model the strand in the cable and will see if it is possible to place a strand in such a way that it would see a significantly lower magnetic field than the other strands, which find themselves at the peak field. And if so - how much would be the difference in terms of strand current, assuming that the total voltage along the length would be the same for all strands. This in effect is equivalent to the assumption that the resistances between the strands inside the joint are low.

Our simplified model of a strand in the cable simulates two last cabling operations. So, the strand represents a spiral with a twist pitch of $160 \mathrm{~mm}$ with a radius of $6.5 \mathrm{~mm}$, which is twisted in a spiral with the central line to be at a radius of $12.5 \mathrm{~mm}$ and with a twist pitch of $450 \mathrm{~mm}$. Fig. 23 shows a cross section of the cable and strand spirals on the periphery of the subcable space, while subcable spirals around center of the symmetry.

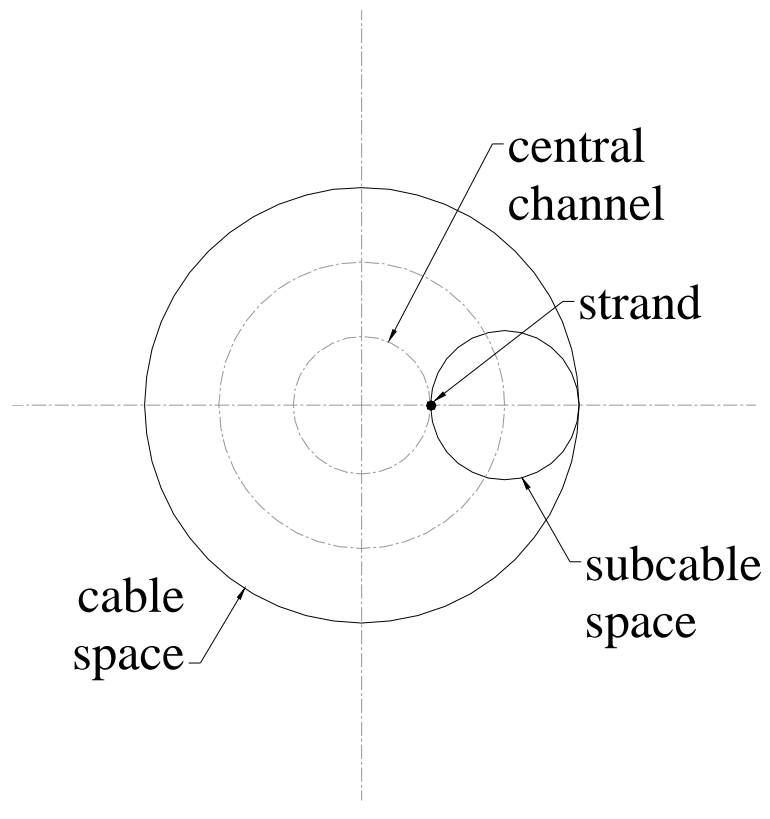

Fig. 23. Model of the strand in the CICC.

To simulate all possibilities of the strands, we calculate magnetic field and electrical field for five strands, which are shifted by $1 / 5$ of the last subcable twist pitch, which is denominated as "shift" in figures. Thus, five shifts make a full period and cover more or less all possibilities for strands location.

Fig. 24 represents results of the local magnetic and electrical field for all of these 5 probe strands on the length of $7.5 \mathrm{~m}$, which contributes the most into the voltage.

As it seen from Fig. 24, the twist pitch of the cable are short enough, or in other words the localization of the magnetic field in the TFMC is not that severe in comparison with strand particular positions, that it can not create very significant difference in conditions in strand despite of their locations.

Fig. 25 shows an average and peak electrical field in the cable over $7.5 \mathrm{~m}$ of the conductor, which contributes the most into the overall voltage. We see that average electrical field (and therefore voltage) varies between strands by $10 \%$, which is insignificant for any noticeable current nonuniformity). We also checked the electric field in the strands, which belong to other subcables and discovered that their voltages are about the same, so the nonuniformity of the magnetic field is not localized enough to cause nonuniform current distribution. 
However, the distribution of local electrical field along the strands is very nonuniform as shown in Fig. 26. This is a known phenomenon. A similar nonuniformity was calculated by A. Nijhuis [7], N.Martovetsky [2] and E. Zapretilina [9]. This nonuniform distribution may represent a threat to the operating conditions of the CICC in the sense that the peak electrical field represents the CICC limitation, not average field if $\mathrm{N}$-value is too high, so the quench will happen with no "warning" in a form of a detectable voltage.

It is important to note that when we see $200 \mu \mathrm{V}$ over the TFMC conductor, the peak electrical field must be very high, higher if the $\mathrm{N}$-value is high. Let's make an estimate how high is the peak electrical field at the moment of take off based on our earlier calculations in this memo. For. N=10, from Fig. 25 we can see that the peak field of $110 \mu \mathrm{V} / \mathrm{m}$ corresponds to average field of about $3 \mu \mathrm{V} / \mathrm{m}$ over the length of $7.5 \mathrm{~m}$, or total voltage of $25 \mu \mathrm{V}$. That means that at the moment of take off at $250 \mu \mathrm{V}$, the peak electrical field must have been about $1100 \mu \mathrm{V} / \mathrm{m}$. This is very high electrical field and normally cannot be reached on an individual NbSn strands with $\mathrm{N}=20-30$ at high currents. That suggests that the CICC strands could not possibly have had $\mathrm{N}=20-30$, since at about $200 \mu \mathrm{V}$ of stable operation observed at experiment, the local electrical field would be several times higher than $1100 \mu \mathrm{V} / \mathrm{m}$, which is incredibly high for NbSn strands at 111 A per strand. This consideration indirectly indicates, that the N-value in the CICC must have been low, much lower than in the original strands with $\mathrm{N}=20-30$. This low $\mathrm{N}$-value in fact helps the measurements during TFMC tests. At high N-values the CICC diagnostics becomes difficult, since the voltages of the run away becomes very small and signal to noise ratio low. Thus, lower $\mathrm{N}$-value, reduces operating margin of the CICC, but improves stability and diagnostics of the CICC conditions.

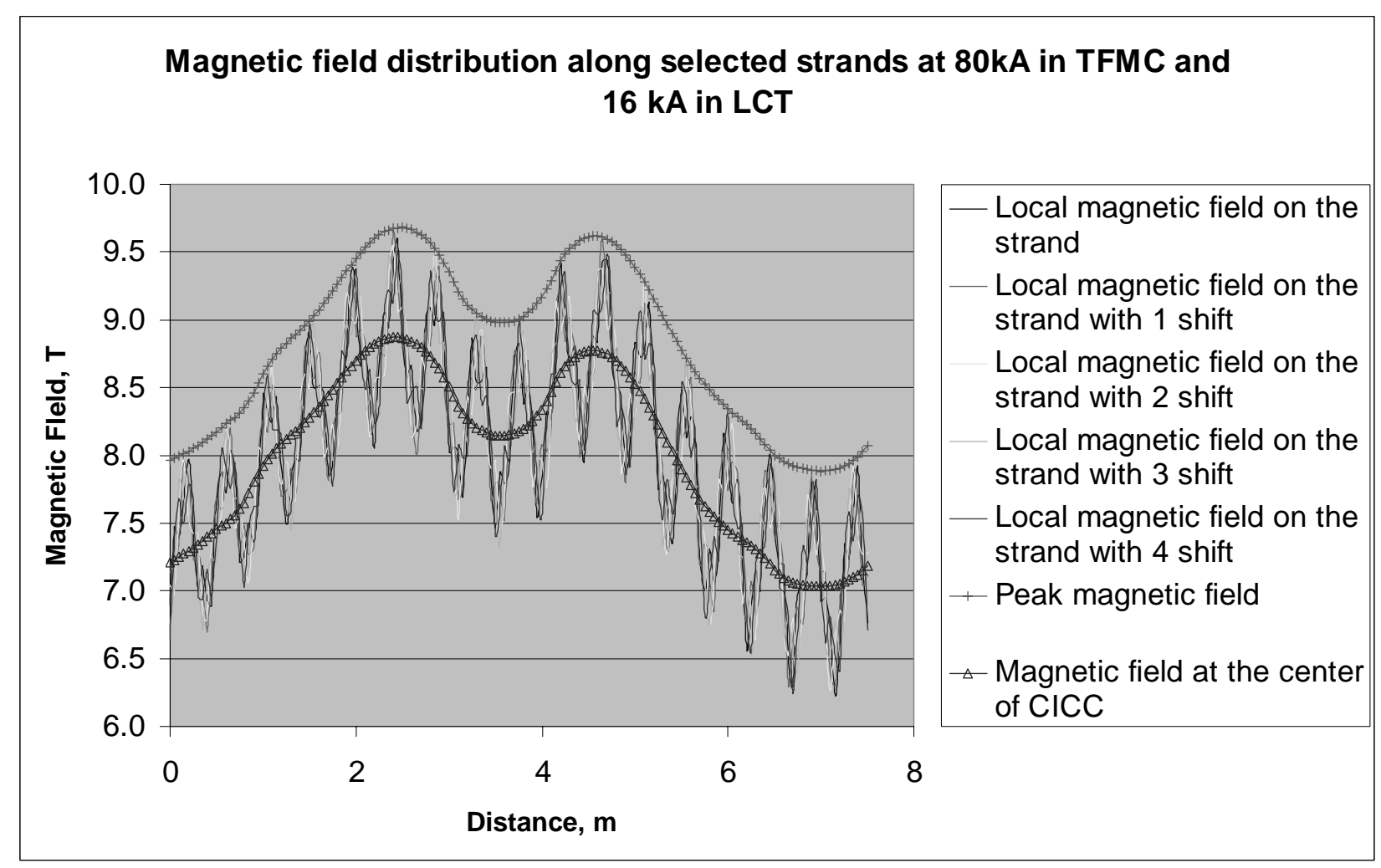

Fig. 24. Distribution of local magnetic field in the strands 


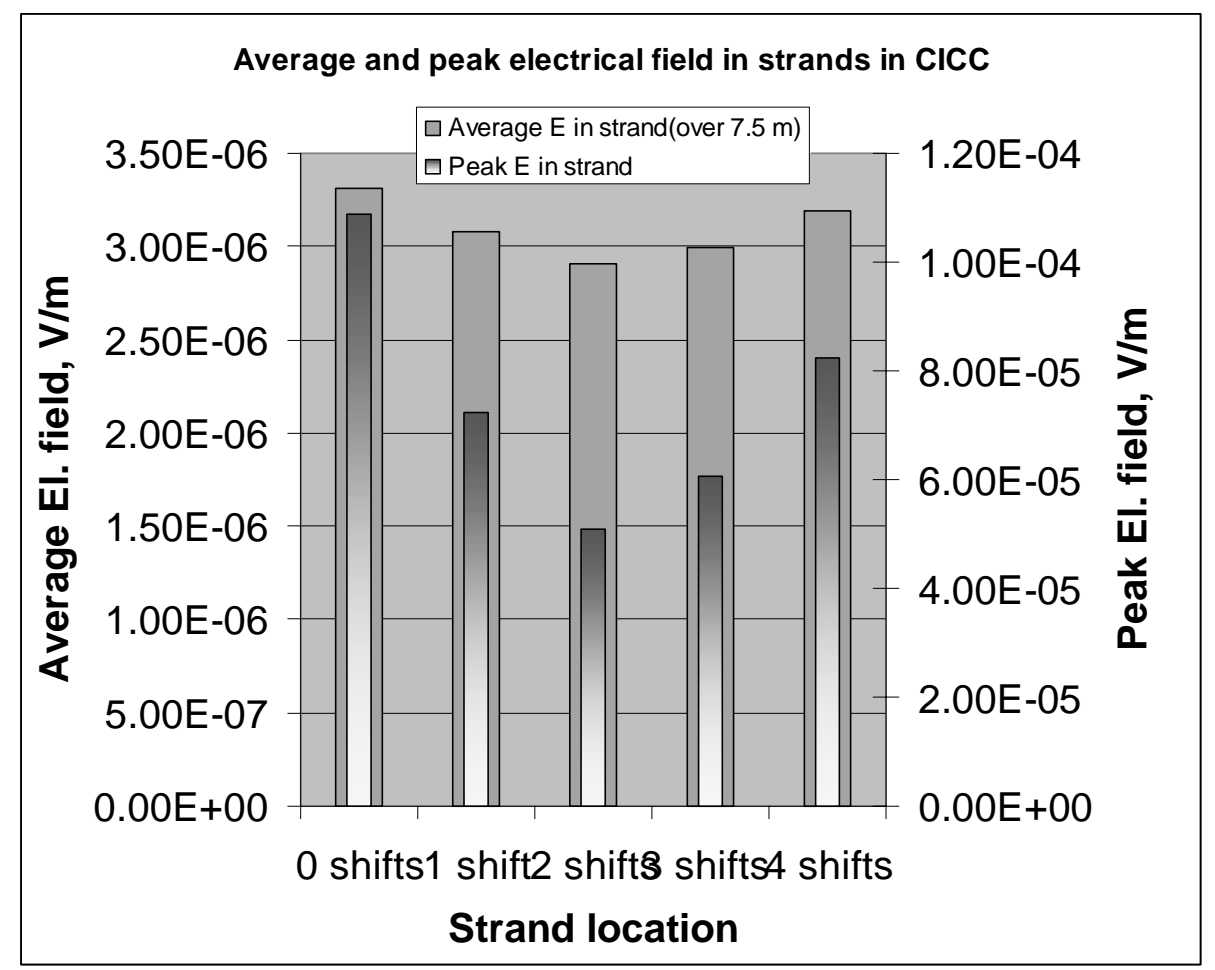

Fig. 25. Average and peak electrical field along the strands in the cable $(\mathrm{N}=10)$

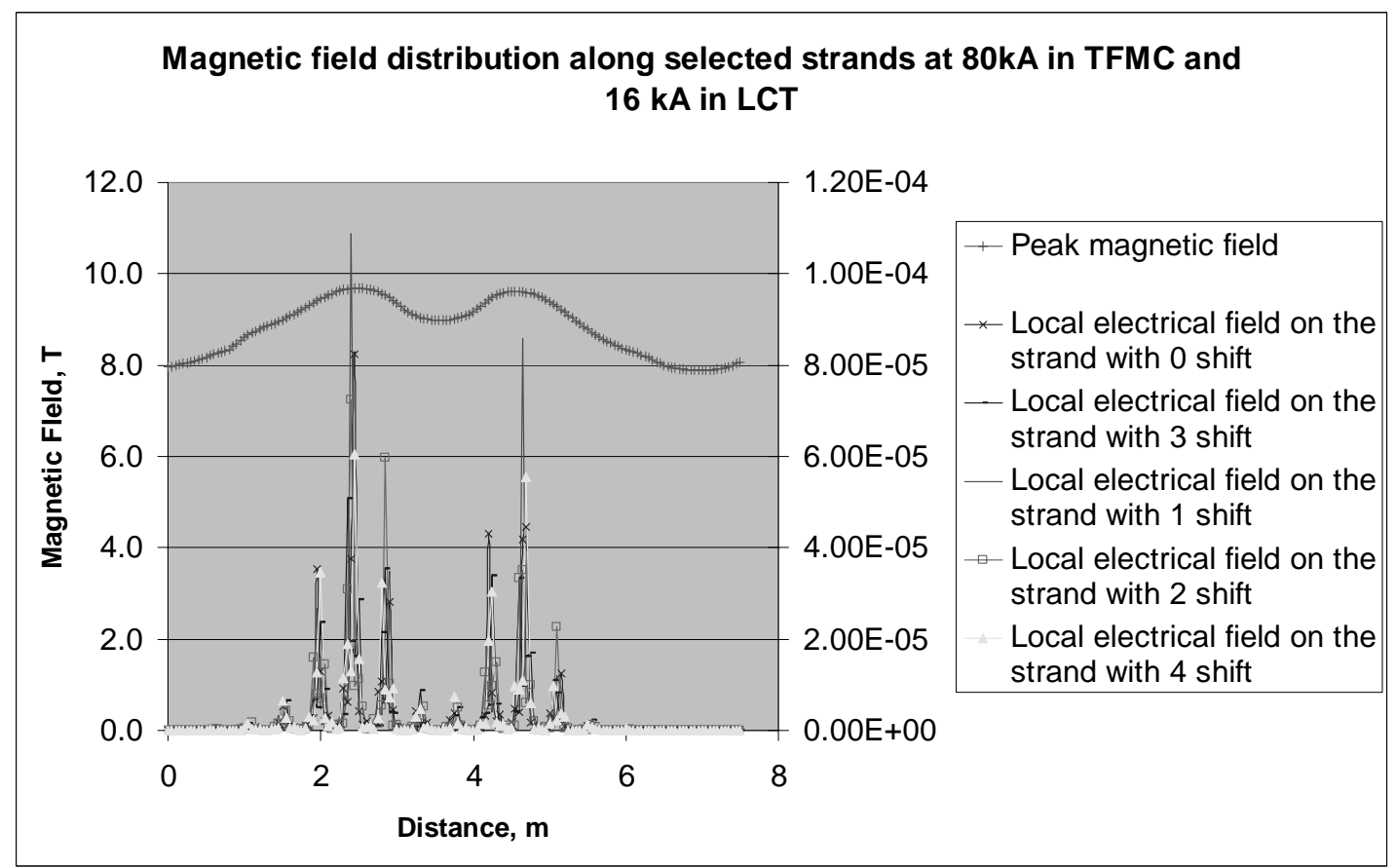

Fig. 26. Electrical field in strands $(\mathrm{N}=10)$

As a result of this study about nonuniformity of the magnetic field in the TFMC we can make the following conclusions:

1. Despite strong nonuniformity of the magnetic field in the TFMC, the twist pitches in the TFMC are short enough to expose all of the strands in the cable to approximately equal conditions, so 
their integrated voltages are approximately equal at equal currents in the strands, which suggests that the current distribution is uniform in the cable within few percent.

2. The fact that the electrical voltage across the TFMC is measured by several hundred micro Volts before take off makes it very unlikely that the N-value could be high in the range of 2030 , because that would mean unsustainably high local electrical fields in the strands.

\section{How valid is the assumption about additional strain causing lower performance than expected? Speculations about the reasons for degradation}

There is a need to develop a predictive tool for CICC degradation for design purposes, even if it is questionable and not very solid scientifically, there is an obvious usefulness of that for practical purposes. Introduction of an additional strain model may be a logical working hypothesis. In this model we assume that there is an additional strain of unidentified origin that we need to add into the original strand correlation to accurately predict the strand properties in the CICC.

Lately, in some studies the explanation to the degradation in CICC in comparison with strand were explained by this "additional strain" which was serving as fitting parameter to explain the CSMC and TFMC test data. There are some reasons to suspect that the degradation observed on the NbSn CSMC inserts, CRPP samples are of mechanical origin:

1. Low strain sensitivity $\mathrm{NbAl}$ did not show any degradation in the CICC tests in the $\mathrm{NbAl}$ Insert test campaign [11].

2. There are experimental studies [12] showing that the lateral force can significantly degrade performance of the CICC, the sensitivity to lateral stress is much worse (stronger) than to the longitudinal stress. Since ITER cables experience very high lateral forces, it is natural to involve this effect into explanation of degradation.

3. Stress of cable in conduit is not very certain, the cable is not that well-defined solid body with well-defined coupling with the conduit and there is a natural tendency to assign all uncertainties to that factor.

4. Some new theories of EM loading effect on the CICC performance or renewed old theories that trying to explain degradation in large CICC $[13,14]$ tend to bring the degradation under "additional" strain hypothesis.

5. CRPP test results on the CICC subscale samples revealed mechanical effects of the EM loads and fabrication on the cable properties [19].

Let us call the hypothesis with the additional strain the EPS model to emphasize the strain factor.

Another way of thinking about the degradation is to assume that the strain is as we think it is from the subscale and strand tests, about $-0.65 \%$, but the critical current is changed, also most likely due to mechanical reasons but in a different way. There must be more than one hypothesis to that, but one of the possibilities is the cracking of the superconducting layer during fabrication, cooldown and/or charging with current and corresponding electromagnetic forces. If one assumes micro cracking in the NbSn layers, there is no reason to assume that the thermodynamic properties like $\mathrm{Tc} 0$ and $\mathrm{Bc} 0$ changed. But transport properties (Ic) may change. Recent experiments by P. Lee with about $0.5 \%$ bending strain and observation of physical cracks [15] may substantiate such a model, which basically reduces Co rather than acts like an additional strain in the Summers correlation. The properties of the CS Insert [16] are better described by this theory, since $\mathrm{Tc}(\mathrm{B}=0 \mathrm{~T})$ of the CS Insert coincides with the $\mathrm{Tc}$ of the strand, thus ruling out "additional" strain due to cooldown or fabrication. Also, the TF Insert properties are better described by the Ic degradation due to Co reduction [17], not due to additional strain, although there is some apparent drop in the Tc $(0 \mathrm{~T})$, which could not be explained completely by this model. We will call this model Co model.

Both mechanisms (additional strain or creation of micro cracking) would cause broader transition to normal state, or in other words reduce $\mathrm{N}$-value and both mechanisms are strain related, but the correlation is somewhat different. The limited amount of data about effect of the stress on the N-value [2] cannot offer large enough drop in $\mathrm{N}$-value to explain the transformation from original strand to a strand in CICC. Crack growth may explain the data on degradation of the CS Insert properties and the CRPP samples [18] during cycles qualitatively, but there are no systematic studies on that issue.

A crucial experiment, which could reveal the difference between these two models, could be a Tc(0T) measurement at low currents at different strains. If Tc is not affected in the CICC in comparison with the 
strand under expected strain, then it may be more appropriate to qualify as a Co degradation. If affected may be "additional" strain correction is a better model to reflect changes.

As a basis of our comparison of these correlations we will use the Summers correlation [5] with the CEA parameters: $\mathrm{C} 0=11000, \mathrm{Bc} 0=29 \mathrm{~T}$ and $\mathrm{Tc} 0=16.9 \mathrm{~K}$ and we will try to match the test data, including the Tc measurement of the TFMC during the re-cooldown campaign in September 2001 at 360 A, where the Tc was measured to be about 16.3-16.5 K).

As a reference point for fitting we use the 80kA, $16 \mathrm{kA}$ run (currents in the TFMC and the LCT, respectively), which yielded $6.12 \mathrm{~K}$ at $111 \mathrm{~A}$ per strand and 9.13T effective field (see our analysis above for $\mathrm{N}=10$ ).

This gives the cooldown strain of $-0.78 \%$ at EM strain of $0.094 \%$. Then, using the CEA correlation with this cooldown strain and adding the EM tensile strain we have the operating strain and can calculate the Tcs for other runs, which we should have expected in the tests according to this EPS model. The assumed EM strain for each run is shown in Table 1.

For the Co model we postulate that the cooldown strain was $-0.65 \%$, based on the full bonded model and results by Specking and Duchateau [10] and then find a Co coefficient, which fits the test result at 80kA and $16 \mathrm{kA}$ in the TFMC and LCT, respectively. Thus, in this model we account for the Ic degradation not by some additional strain, but by the reduction in the Co coefficient.

Fig. 27 shows results of such a comparison in terms of current sharing temperatures in the TFMC tests and Table 1 gives the parameter values for comparison between the two models. The first two columns in Table 1 show transport currents in the LCT and in the TFMC, respectively. B eff shows effective magnetic field defined above for $\mathrm{N}=10$, epsEM is the assumed electromagnetic strain and eps cd - cooldown strain before EM loading.

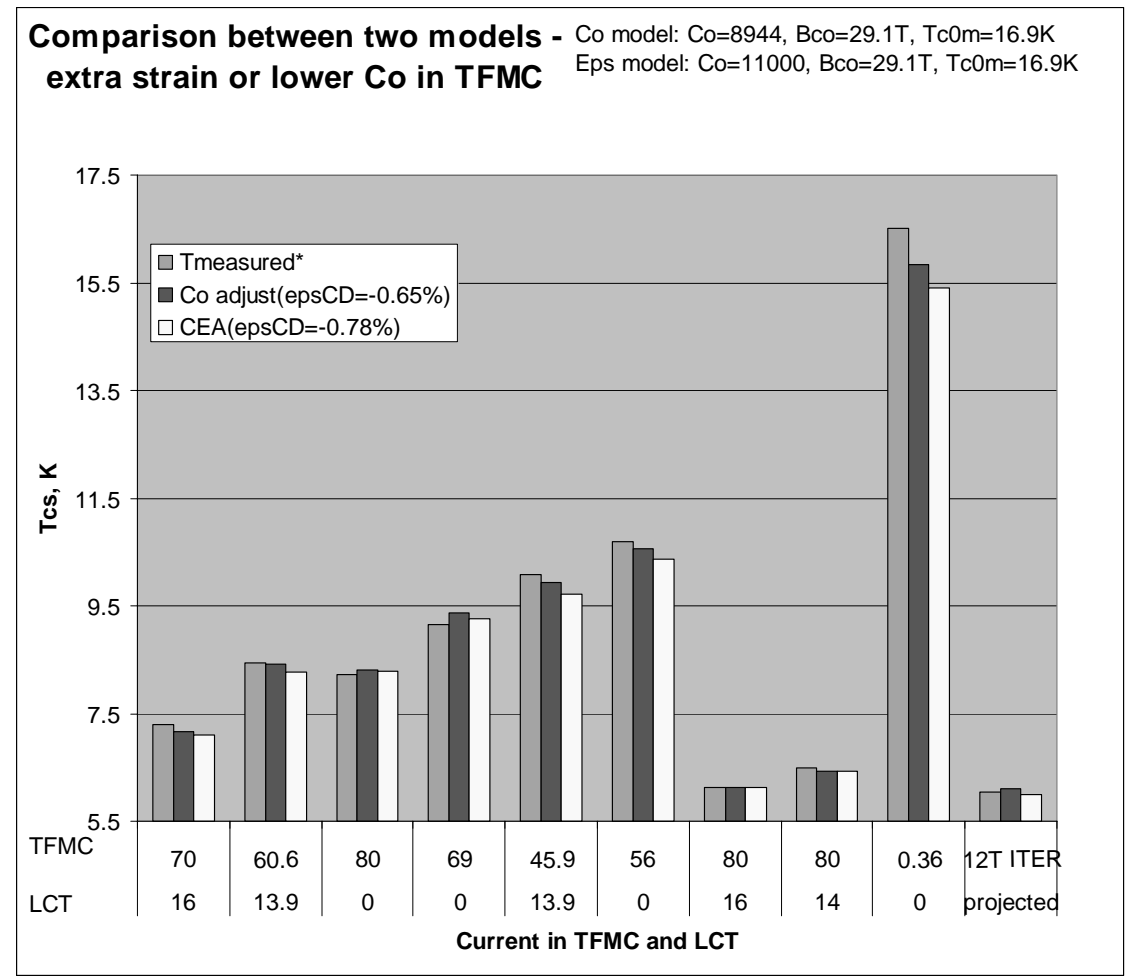

Fig. 27. Comparison between the two models and the measured Tcs. 12 T ITER operation is projected to satisfy $1 \mathrm{~K}$ margin for EPS model.

Table 1. Assumed parameters for the model comparisons and calculation results. 


\begin{tabular}{|c|c|c|c|c|c|c|c|c|c|c|c|c|c|}
\hline $\begin{array}{l}\text { LCT current, } \\
\text { kA }\end{array}$ & $\begin{array}{l}\text { TFMC } \\
\text { current, kA }\end{array}$ & $\begin{array}{l}\text { Tmeasure } \\
d^{*}\end{array}$ & I A/strand & Beff, T & epsEM & $\begin{array}{l}\text { eps } \\
\text { cd(EPS) }\end{array}$ & $\begin{array}{l}\text { eps } \\
\text { oper(Eps } \\
\text { model) }\end{array}$ & $\begin{array}{l}\text { eps } \\
\text { cd(Co) }\end{array}$ & $\begin{array}{l}\text { eps oper(Co } \\
\text { model) }\end{array}$ & $\begin{array}{l}\text { Tcs for } \\
\text { Co } \\
\text { adjust(ep } \\
\text { sCD=- } \\
0.65 \%)\end{array}$ & $\begin{array}{l}\text { Tcs for } \\
\text { CEA(eps } \\
\text { CD=- } \\
0.78 \%)\end{array}$ & $\begin{array}{l}\text { Co model, } \\
\text { DT, K } \\
\text { measured- } \\
\text { predicted }\end{array}$ & $\begin{array}{l}\text { Eps } \\
\text { I, model, } \\
\text { DT, K } \\
\text { d-measured- } \\
\text { predicted }\end{array}$ \\
\hline 16 & 70 & 7.3 & 97.22 & 8.4 & $0.074 \%$ & $-0.778 \%$ & $-0.705 \%$ & $-0.650 \%$ & $-0.577 \%$ & $\quad 7.17$ & 7.09 & 0.125 & $\begin{array}{ll}5 & 0.207\end{array}$ \\
\hline 13.9 & 60.6 & 8.43 & 84.17 & 7.32 & $0.055 \%$ & $-0.778 \%$ & $-0.723 \%$ & $-0.650 \%$ & $-0.595 \%$ & 8.42 & 8.27 & 0.010 & 0.159 \\
\hline 0 & 80 & 8.225 & 111 & 6.7 & $0.080 \%$ & $-0.778 \%$ & $-0.698 \%$ & $-0.650 \%$ & $-0.570 \%$ & 8.32 & 8.28 & -0.095 & -0.056 \\
\hline 0 & 69 & 9.15 & 95.83 & 5.8 & $0.060 \%$ & $-0.778 \%$ & $-0.718 \%$ & $-0.650 \%$ & $-0.590 \%$ & 9.37 & 9.27 & -0.223 & -0.116 \\
\hline 13.9 & 45.9 & 10.075 & 63.75 & 6 & $0.033 \%$ & $-0.778 \%$ & $-0.745 \%$ & $-0.650 \%$ & $-0.617 \%$ & 9.93 & 9.71 & 0.141 & 0.366 \\
\hline 0 & 56 & 10.7 & 77.78 & 4.75 & $0.039 \%$ & $-0.778 \%$ & $-0.739 \%$ & $-0.650 \%$ & $-0.611 \%$ & 10.55 & 10.38 & 0.151 & 0.325 \\
\hline 16 & 80 & 6.12 & 111.11 & 9.13 & $0.094 \%$ & $-0.778 \%$ & $-0.684 \%$ & $-0.650 \%$ & $-0.556 \%$ & 6.12 & 6.12 & 0.000 & 0.000 \\
\hline 14 & 80 & 6.5 & 111.11 & 8.8 & $0.092 \%$ & $-0.778 \%$ & $-0.686 \%$ & $-0.650 \%$ & $-0.558 \%$ & 6.44 & 6.44 & 0.060 & 0.061 \\
\hline 0 & 0.36 & 16.5 & 0.5 & 0.01 & $0.000 \%$ & $-0.778 \%$ & $-0.778 \%$ & $-0.650 \%$ & $-0.650 \%$ & 15.84 & 15.40 & 0.660 & 1.100 \\
\hline projected & 12T ITER & 6.05 & 69 & 11.5 & $0.20 \%$ & $-0.778 \%$ & $-0.578 \%$ & $-0.650 \%$ & $-0.450 \%$ & 6.10 & 6.00 & -0.050 & 0.050 \\
\hline
\end{tabular}

The projected ITER operation corresponds to $1 \mathrm{~K}$ margin for EPS model and corresponds to $69 \mathrm{~A}$, and for this particular point the blue column in Fig. 27 does not represent "measured", since it was not actually measured in the TFMC campaign, it is just a mean value between the two models prediction.

Looking at Fig.27 one may conclude that the Co model fits measured data a little better than the EPS model. This is not a proof, that the Co is closer to reality yet, but at least it shows that the additional strain theory is not the only possible explanation to observed facts and also that the Co degradation theory may be more or at least as useful as EPS theory for designing ITER and other magnets with CICC. But the difference at $12 \mathrm{~T}$ projected from $9 \mathrm{~T}$ operation from both models is small. In other words, we may conclude that even though the TFMC did not reach exactly $12 \mathrm{~T}$ operation, it was close enough to make projections to $12 \mathrm{~T}$ comfortably accurate, which emphasizes a great value of the TFMC test data for $12 \mathrm{~T}$ ITER operation.

It is interesting to look retrospectively at our expectations before the TFMC test campaign and compare it with the test results.

The pretest analysis [20] assumes the cooldown strain of $-0.61 \%$ with the EM negligible, according to this reference. Later it was corrected that the strain due to EM loading was not so negligible, but let's suppose it was for this exercise. Then, as a rough model we may expect conservatively, that we design the magnet using peak magnetic field and expect no degradation from other reasons. Using CEA correlation, we use $9.7 \mathrm{~T}$ peak field and $-0.61 \%$ cooldown strain and no credit to the EM strain. Then, the prediction for the Tcs would be $6.1 \mathrm{~K}$, which is very close to the measured value. That assessment would have suggested that the CICC behaves exactly as expected, no degradation is observed. In effect, the hidden safety factor when we assumed that all the strands are sitting in the peak field and are not strained by the EM forces and the Tcs for the CICC is defined at much higher level of electrical field than for a strand would be balanced by degradation. If we do not go into details, we may draw a conclusion that taking such an approach for designing the magnet is adequate and accurate enough. While it may be adequate for the design purposes in some favorable circumstances, it would have never revealed the nature of the strand properties transformation in the CICC. The issue of comparison can become even more confusing if the take off temperature at the pancake inlet were compared to the strand Tcs expectations. In that case a wrong conclusion could be made, that the CICC exceeded the strand performance. But if such properties would be used for design of a magnet where several tens of meters are exposed to a high magnetic field, these properties could not have been reached, since the total self-heating would have been unsustainable. This joggling of the arguments shows that developing expectation of the CICC performance from the strand properties is a complicated process involving many parameters and can be easily confusing if hidden safety margins are not revealed and realized.

Comparison in terms of difference between the model prediction and the measured values is shown in Fig. 28, which uses the same data as Fig.27.

It shows a little more clearly that the Co model is in general closer to the test results, except insignificant differences of about $0.1 \mathrm{~K}$ in two cases. Difference at $80 \mathrm{kA}$ in the TFMC and $16 \mathrm{kA}$ in the LCT is zero, because they were used as fitting points and matched precisely. The point for Tc (0T) is especially interesting. It is significantly better for the Co model than the one for the EPS model, which suggests two possibilities: 1) the $16.9 \mathrm{~K} \mathrm{Tc}$ in the correlation is not good for describing properties at zero B and I and/or 2 ) the real cooldown strain is significantly less in the strands than $-0.75-0.78 \%$ as the EPS model suggests. Since we do not have reliable data on the strand properties in this area versus strain, an explanation remains to be found. 


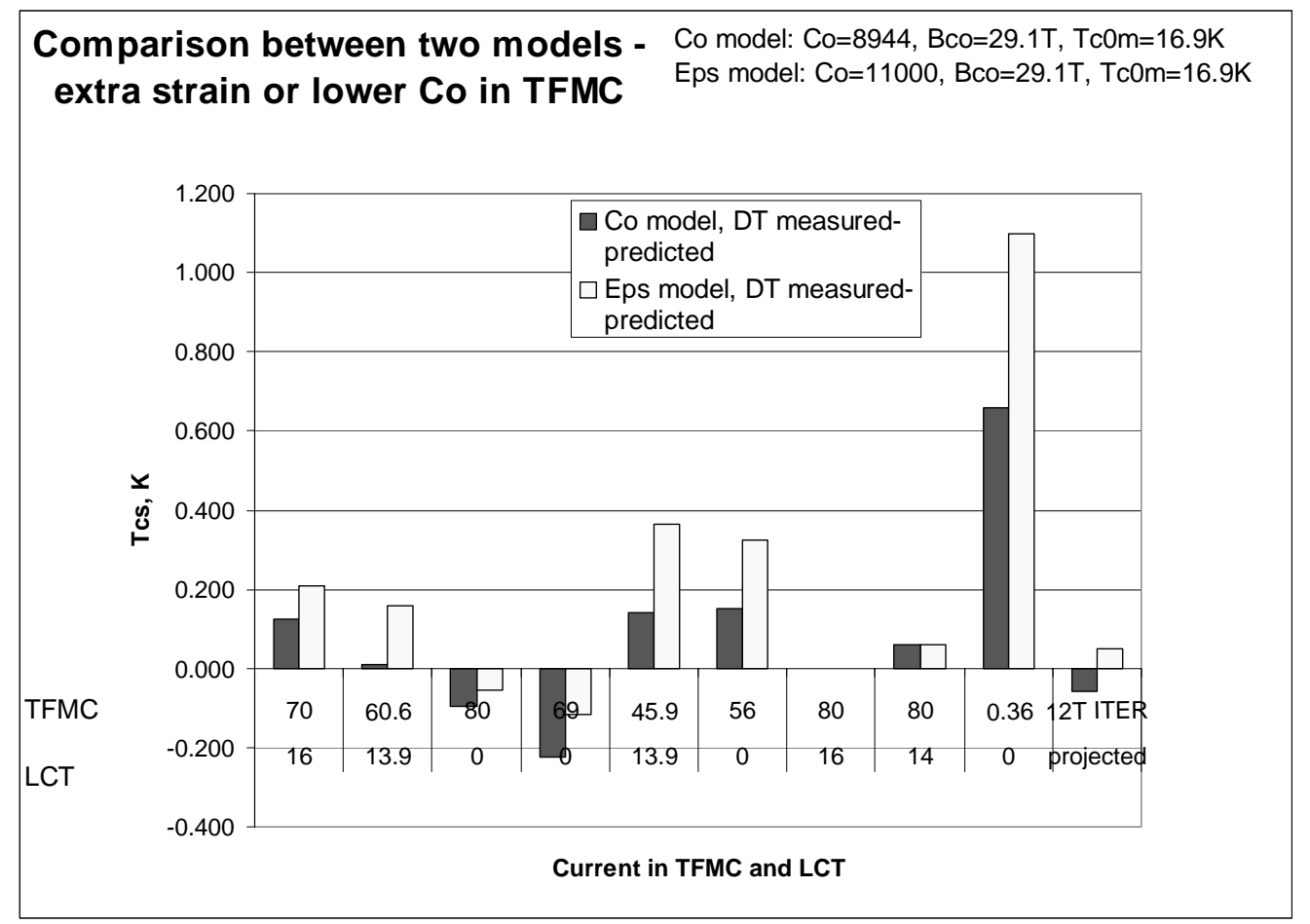

Fig. 28. Comparison between EPC and Co models with the test data.

The Co model for the TFMC assumes a factor of 0.81 degradation in the Co parameter, which compares with the Co degradation of the CS Insert before cycling as follows: the CS Insert degradation was 0.78 to 0.61 depending on the strand Ic assumptions; 0.78 if QA Ic data are used and CS Insert properties are taken before cycles, 0.61 if strand witness Ic is used, and also less than what was observed on the TF Insert, where the Ic was about 0.6 of expected value. In both cases of comparison for the CS Insert and the TF Insert we assumed that the strain of the strand in the CICC is about the same as for the strand measured on the ITER barrel due to the combination of a little higher compression in cooldown but some tensile strain from the EM loading. Such an observation suggests that all large NbSn CICC tested in the ITER Model Coil programs experienced noticeable Ic reduction in comparison with expected values, but the amount of reduction varied significantly between the experiments with the TFMC having the lowest reduction in comparison with expected values. Here we use word "reduction" to distinguish the portion of degradation which was expected from the CTE mismatch from the unexpected portion of degradation. The degradation is defined in this memo as reduction of the critical current or current sharing temperature at a given current in comparison with the strand under the same strain, local coolant temperature, effective magnetic field, etc at the "critical" electrical field of $10 \mu \mathrm{V} / \mathrm{m}$. In other words, degradation includes all factors preventing realization of the strand potential in the CICC, reversible and irreversible.

The low CTE conduits in the CS Insert and the TF Insert still result in lower loss of the strand performance (or lower overall degradation) in the CICC than in the TFMC. In other words, it is more beneficial to use low CTE conduit even though the advantage is not as strong as formal CTE mismatch and corresponding strain may suggest. Fig. 29 shows comparison between the NbSn strands and CICC tested in the ITER Model Coil programs. These curves were calculated for $12 \mathrm{~T}$ performances at $10 \mu \mathrm{V} / \mathrm{m}$ electrical field by correlations, which satisfy test data. All CICC are assumed to experience about $0.1 \%$ strain due to electromagnetic load, which makes the comparison sensible.

It shows that the low CTE conduit (Incoloy for the CS Insert and Ti for the TF Insert) helps realizing the superconducting strand properties better, than the stainless steel conduit in the TFMC, as expected, although expectations for low CTE CICC were even higher. In other words, although stainless steel conduit suppresses superconducting properties more dramatically, the level of predictability for the TFMC is a little better than for the low CTE CICC. Like the TF Insert, the TFMC did not show any sensitivity to the cycles. 


\section{Comparison of strand to conductor performance in CS,TF Inserts and TFMC at $12 \mathrm{~T}, 10 \mathrm{uV} / \mathrm{m}$}

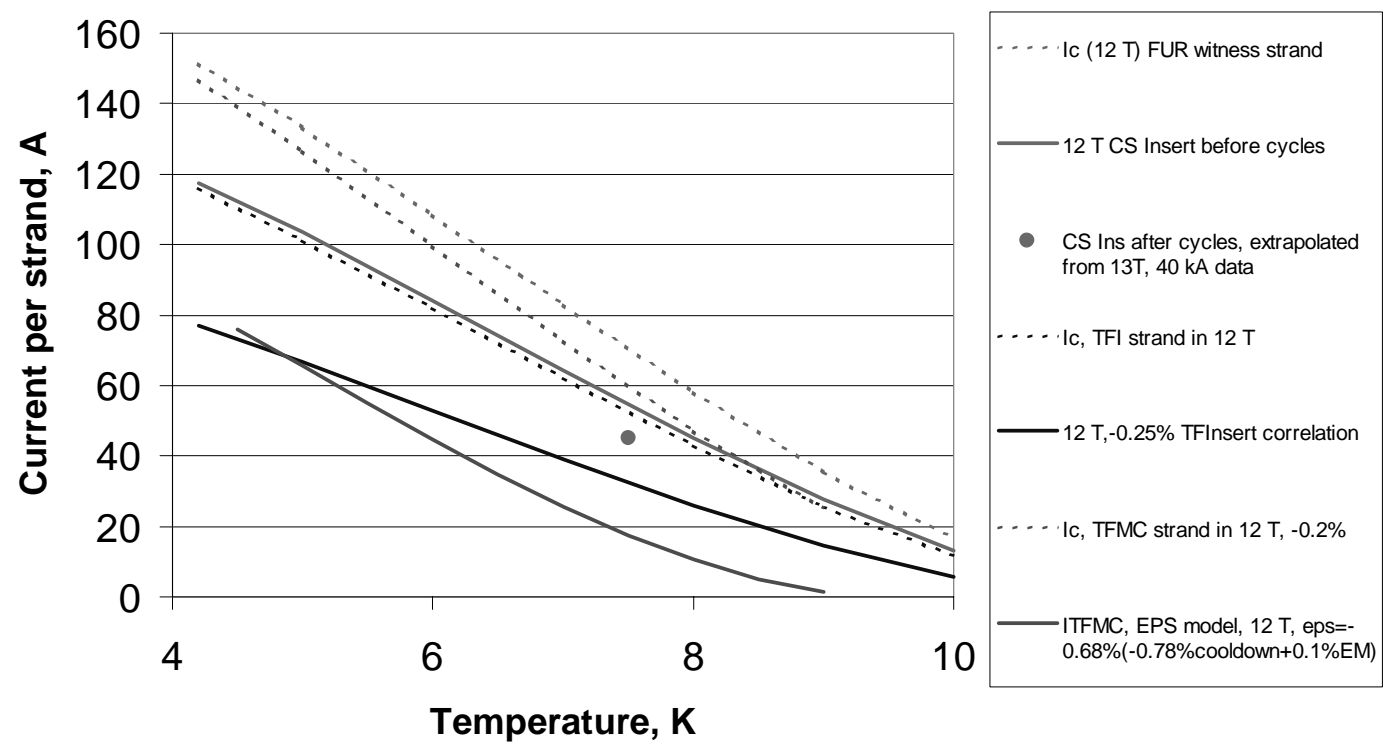

Fig. 29. Comparison between the strand performance and the CICC performance in the NbSn CICC tested in the ITER Model Coil program.

\section{Conclusions}

My assessment of the TFMC test data, comparison with the strand data and other NbSn CICC conductors tested in the ITER R\&D effort suggests the following.

1. Despite some uncertainty in the test data, the TFMC provides valuable information about large CICC behavior, very relevant for the large magnet design.

2. The TFMC shows noticeable degradation in comparison with expectations of the strand performance at the voltage level of $10 \mu \mathrm{V} / \mathrm{m}$.

3. Transition to normal state in the TFMC appears to be significantly broader than expected for the strand under the same conditions (N-value significantly lower than for the LMI strands), although there is a lack of data on the LMI strand under relevant strains, which makes unambiguous conclusion difficult. Broader transition in the CICC and very dissimilar electrical field distribution in the CICC and a strand makes definition of degradation difficult to be expressed quantitatively and requires a significant number of assumptions. Nevertheless, such a comparison is possible and is very relevant and useful for the magnet design.

4. Fitting the test data with the model of "additional strain" to be plugged into the strand original correlation yields relatively accurate approximation for a range of fields in the range of 5-9 T, but is not consistent with the data at zero magnetic field and low currents. The same or even better accuracy for $10 \mu \mathrm{V} / \mathrm{m}$ criteria could be obtained if degradation assumed not due to an "additional strain" but due to the critical current reduction. But without further experimental studies both models remain speculative, although giving close predictions for $12 \mathrm{~T}$ operation.

5. Inhomogeneity of the magnetic field in the TFMC cannot contribute significantly into the nonuniform current distribution; all strands in the cable are approximately equally exposed to the magnetic field. 
6. Discrepancy between measured and expected performance of the TFMC is lower than in the CS Insert and the TF Insert, although comparison between the NbSn CICC proved again that the low CTE conduit allows much better utilization of the superconducting properties.

7. There is a need in deeper understanding of the NbSn strand properties transformations from the stand-alone strand to the strand in the CICC. The empirical models which attempt to describe the change do not reflect the change in the broadness of transition (N-value) and thus have only limited usefulness, although may be sufficient for the practical purposes of magnet design if magnet operating point is not far away from the experimentally verified CICC performance.

\section{References}

1. R.Zanino, L. Savoldi, M\&M analysis of measured and expected/design performance in the TFMC Tcs tests, presentation at the $3^{\text {rd }}$ TFMC Test Group meeting, Karlsruhe, Germany, January 22-23, 2002.

2. N.Martovetsky, memo 5/18/2001, distributed within ITER magnet team, unpublished

3. P.Hertout, internal CEA reports and spreadsheet available on request, private communication.

4. G.L. Dorofeev, A.B. Imenitov, E.Yu. Klimenko, Cryogenics, 1980, v.20, N6, p.307

5. L. T. Summers, M. Guinan et al, A Model for the Prediction of Nb3Sn Critical Current as a Function of Field, Temperature and Radiation Damage, IEEE Trans on Magnetics, 27(2), March 1991, 2041

6. A. Godeke and H. Krooshoop et al, "Wide temperature and field scaling relations in Nb3Sn ITER strands", University of Twente, Final report to NET, September 2000

7. A. Nijhuis and Yuri Ilyin, Current distribution: reconstruction from self-field measurements and impact on cable n-value, Univ. of Twente, Dec. 2001, EDFA-00/552

8. R. Zanino, M. Bagnasco and L.Savoldi Richard, "Preliminary analysis of TFMC Tes tests, phase II", private communication distributed to the TFMC test participants.

9. E.Zapretilina, report at the TFCI meeting at Efremov Inst., March 2002

10. W. Specking, JL Duchateau, First Results of Strain Effects on Critical Current of Incoloy Jacketed $\mathrm{Nb}_{3}$ Sn CICCs, Proceedings 15th Int Conf on Mag Tech, Beijing, Oct, 1997

11. K. Okuno, et al, Test of the NbAl Insert and ITER Central Solenoid Model Coil, presented at the ASC 2002, Houston.

12. L.T. Summers and J.R. Miller, Further Studies of Transverse Stress Effects in Cable-InConduit Conductors, Adv. Cryo. Eng., Vol. 36A, 101, 1990

13. M. Takayasu - presentation at the Meeting on Test Results of the CS Model Coil and the CS Insert, Nov.9-11, 2000, Naka, Japan, unpublished

14. N. Mitchell, Presentation at US-Japan Workshop on Superconducting Magnet Technology, 1215 August, 2002

15. P.Lee, Presentation at US-Japan Workshop on Superconducting Magnet Technology, 12-15 August, 2002

16. N. Martovetsky, P. Michael, J. Minervini et al, "ITER CS Model Coil and CS Insert test results" IEEE Trans. Appl. Superconduct., vol. 11, N1, pp. 2030-2033, March 2001.See also: T. Kato, H. Tsuji, T. Ando, et al., "First test results for the ITER central solenoid model coil" Fusion Engineering and Design, 56-57 (2001), 59-70

17. N.Martovetsky, Presentation at the meeting on the TF Insert test results, St. Petersburg, March 2003.

18. P.Bruzzone, A. Fuchs, B. Stepanov, G.Vecsey, "Performance evolution of NbSn cable-inconduit conductors under cyclic load", IEEE Trans. Appl. Superconduct., vol. 12, No.1, March 2002, p. 516-519.

19. P.Bruzzone, Summary of CRPP tests on CICC, Presentation at US-Japan Workshop on Superconducting Magnet Technology, 12-15 August, 2002

20. ITER Toroidal Field Model Coil (TFMC) Test and Analysis Summary Report, compiled by P. Libeyre, CEA AIM/NTT-2001.005 